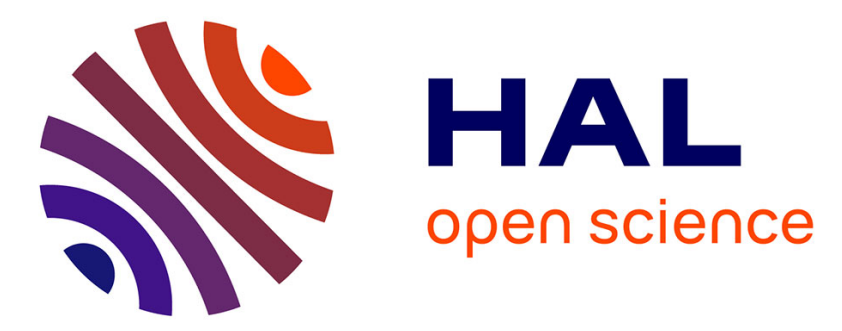

\title{
Totally dissipative measures for the shift and conformal $\sigma$-finite measures for the stable holonomies
}

Renaud Leplaideur

\section{To cite this version:}

Renaud Leplaideur. Totally dissipative measures for the shift and conformal $\sigma$-finite measures for the stable holonomies. Boletim da Sociedade Brasileira de Matemática, 2010. hal-02915272

\section{HAL Id: hal-02915272 \\ https://hal-unc.archives-ouvertes.fr/hal-02915272}

Submitted on 14 Aug 2020

HAL is a multi-disciplinary open access archive for the deposit and dissemination of scientific research documents, whether they are published or not. The documents may come from teaching and research institutions in France or abroad, or from public or private research centers.
L'archive ouverte pluridisciplinaire HAL, est destinée au dépôt et à la diffusion de documents scientifiques de niveau recherche, publiés ou non, émanant des établissements d'enseignement et de recherche français ou étrangers, des laboratoires publics ou privés. 


\title{
Totally dissipative measures for the shift and conformal $\sigma$-finite measures for the stable holonomies
}

\author{
Renaud Leplaideur*
}

\begin{abstract}
In this paper we investigate some results of ergodic theory with infinite measures for a subshift of finite type. We give an explicit way to construct $\sigma$-finite measures which are quasi-invariant by the stable holonomy and equivalent to the conditional measures of some $\sigma$-invariant measure. These $\sigma$-invariant measures are totally dissipative, $\sigma$-finite but satisfy a Birkhoff Ergodic-like Theorem .

The constructions are done for the symbolic case, but can be extended for uniformly hyperbolic flows or diffeomorphisms.

Keywords: holonomy, shift, $\sigma$-finite measure, hole, dotted-system, Gibbs states, return time
\end{abstract}

\section{Introduction and statements of results}

We consider a mixing two sided subshift of finite type with finite alphabet $(\Sigma, \sigma)$ The set of vertices of the defining graph of $(\Sigma, \sigma)$ is $\{1, \ldots, N\}$ with $N \geq 2$. We denote by $\mathcal{A}=\left(a_{i j}\right)$ the $N \times N$-transition (aperiodic) matrix of 0,1 's associated to $\Sigma$; namely points in $\Sigma$ are sequences $x=\left(x_{n}\right)_{n \in \mathbb{Z}}$ such that for every $n, x_{n}$ belongs to $\{1, \ldots, N\}$ and

$$
a_{x_{n} x_{n+1}}=1 \text {. }
$$

In $\Sigma$, the cylinder $\left[i_{k}, \ldots, i_{k+n}\right]$ denotes the set of points $y \in \Sigma$ such that $y_{j}=i_{j}$ (for every $k \leq j \leq k+n$ ). Such a cylinder is also called a word (of length $n+1$ ) or equivalently a $(k, k+n)$-cylinder.

The letter $R$ denotes one of the $(0,0)$-cylinders of $\Sigma$. Throughout we assume that there exists a periodic orbit for $\sigma$ in $\Sigma$ which avoids $R$. Note that unless $\Sigma$ is a unique periodic orbit, there always exists such a periodic orbit as soon as $R$ is chosen small enough. Then, a higher-block representation allows us to consider the cylinder $R$ as a $(0,0)$-cylinder.

Let $\phi$ be a $\alpha$-Hölder continuous function from $\Sigma$ to $\mathbb{R}$. We assume that $\phi$ is dependent only on the future. Let $\beta$ be a real number. Let $m_{\beta}$ be the unique equilibrium state associated to the potential $\phi-\beta \mathbb{1}_{R}$. In [Lep05] we proved that

\footnotetext{
*Laboratoire de Mathématiques, UMR 6205, Université de Bretagne Occidentale, 6 rue Victor Le Gorgeu BP 809 F - 29285 BREST Cedex, Renaud.Leplaideur@univ-brest.fr
} 
$m_{\beta}$ converges to a measure $m$ when $\beta$ goes to $+\infty$. Moreover $m$ is a maximizing measure for $-\mathbb{I}_{R}$ with maximal $\phi$-pressure among these measures. This means that

$$
-m(R)=\max _{\nu-i n v}\{-\nu(R)\}
$$

and $h_{m}(\sigma)+\int \phi d m=\max \left\{h_{m^{\prime}}(\sigma)+\int \phi d m^{\prime}\right\}$, where the maximum is taken over measures $m^{\prime}$ satisfying

$$
-m^{\prime}(R)=\max _{\nu \text { a-inv }}\{-\nu(R)\} .
$$

Due to our assumption on $R$, we get here

$$
\max _{\nu \rightarrow-i n v}\{-\nu(R)\}=0 .
$$

In [Lep00] we introduced a method to study the local structure of the Gibbs measure for the system $(\Sigma, \sigma)$ associated to the potential $\phi$. The main points are recalled in Section 2. Let $g$ be the first return map into $R$ by iterations of $\sigma$. We prove here (see Lemma 2.1) that a direct consequence of the construction in [Lep00] is that there exists a $g$-invariant measure $\check{\mu}_{\beta}$ in $R$ such that

$$
\check{\mu}_{\beta}(.)=\frac{m_{\beta}(. \cap R)}{m_{\beta}(R)} .
$$

The main motivation for this paper was then to investigate what happens when the parameter $\beta$ goes to $+\infty$. Remember that $\lim _{\beta \rightarrow+\infty} m_{\beta}(R)=0$. Our goal was to understand how the connection between $\check{\mu}_{\beta}$ and $m_{\beta}$ given by (1) breaks off: does $\check{\mu}_{\beta}$ also converges and/or if $\widehat{\mu}$ is an accumulation point for $\check{\mu}_{\beta}$ as $\beta$ tends to $+\infty$ is there still a relation between $m_{\infty}$ and $\widehat{\mu}$ ?

Our first result is:

\section{Theorem 1 With these notations,}

1. $\check{\mu}_{\beta}$ converges to some probability measure $\widehat{\mu}$ with support in $R$ when $\beta$ goes to $+\infty$.

2. The support of $\widehat{\mu}$, satisfies supp $\widehat{\mu} \cap \sigma^{k}($ supp $\widehat{\mu})=\emptyset$ for every $k \neq 0$.

3. the opened-out measure $\mu^{\prime} \stackrel{\text { def }}{=} \sum_{k \geq 0} \sigma_{*}^{k} \widehat{\mu}$ is a $\sigma$-finite measure with the same asymptotic as that of $m$ : for $\mu^{\prime}$ almost every $x$ and for every continuous function $\psi$,

$$
\lim _{n \rightarrow+\infty} \frac{1}{n} S_{n}(\psi)(x)=\int \psi d m_{x}
$$

where $m_{x}$ is one of the ergodic components of $m$.

Remark 1. The explanation to consider $\check{a}$ and $\widehat{a}$ will appear soon.

We emphasize that (2) says that there is still some memory of the connection at the limit. We want to insist on this last point. Even if the measure $\mu^{\prime}$ is totally dissipative it has some dynamical asymptotic given by (2). Note that usually, 
dissipative measures are not studied because typical points are not recurrent (from the measure point of view). Moreover we emphasize that the property (2) is very different from the conservative case. Indeed, for the conservative case on compact set, continuous functions are not in $L^{1}$. It is also known (see [Aar97]) that there are no constants $a_{n}>0$ such that almost everywhere and for every function in $L^{1}$

$$
\frac{S_{n}(f)}{a_{n}} \rightarrow_{n \rightarrow+\infty} \int f
$$

On the contrary, in our case, we deal with continuous functions and have convergence or the usual Birkhoff average.

It turns out that our result is related to the question of the unique ergodicity for horocycle foliations. This is the second motivation for this paper. This question was studied by R. Bowen and B. Marcus in [BM77], by N. Haydn in [Hay94] and by the author in [Lep00] (among others). We shall first introduce some notations and vocabulary.

For $x=\left(x_{n}\right)$ in $\Sigma$ we denote by $W_{l o c}^{u}(x)$ the local unstable leaf at $x$ :

$$
y=\left(y_{n}\right) \in W_{l o c}^{u}(x) \Longleftrightarrow \forall n \leq 0, y_{n}=x_{n} .
$$

The global unstable leaf $W^{u}(x)$ is defined by

$$
W^{u}(x)=\bigcup_{n \geq 0} \sigma^{n}\left(W_{l o c}^{u}\left(\sigma^{-n}(x)\right)\right)
$$

This corresponds to the set of points $y$ whose past eventually equals the past of $x$. Local and global stable leaves are defined similarly exchanging the forward and the backward directions. The set of local unstable leaves is a measurable partition (see [Roh62]).

Let $x$ and $y$ be two points in $\Sigma$. Let $K_{x}$ and $K_{y}$ be two compact sets respectively in $W_{l o c}^{u}(x)$ and $W_{l o c}^{u}(y)$. A stable holonomy from $K_{x}$ onto $K_{y}$ is an invertible map $h^{s}: K_{x} \rightarrow K_{y}$ such that for every $z$ in $K_{x}, h^{s}(z)$ belongs to $K_{y} \cap W^{s}(z)$.

Let $\tau_{\phi}$ denotes the Gibbs measure for the system $(\Sigma, \sigma)$ associated to the potential $\phi$ (i.e. $\left.\tau_{\phi}=m_{0}\right)$. Let $\left(\tau_{\phi, x}^{u}\right)$ denotes the system of conditional measures of $\tau_{\phi}$ with respect to the partition in local unstable leaves. There exists a system of measures $\left(\nu_{\phi, x}^{u}\right)$ such that

(H1) $\nu_{\phi, x}^{u}$ is a probability measure equivalent to $\tau_{\phi, x}^{u}$,

(H2) For every $x$ and $y$ in $\Sigma$, for every stable holonomy $h^{s}: K_{x} \rightarrow K_{y}$ and for every $z$ in $K_{x}$

$$
\frac{d \nu_{\phi, y}^{u} \circ h}{d \nu_{\phi, x}^{u}}=e^{\omega(z, h(z))},
$$

where $\omega(z, h(z))=\sum_{j=0}^{+\infty}\left[\phi \circ \sigma^{j}(z)-\phi \circ \sigma^{j} \circ h(z)\right]$.

For the rest of the paper, a system of measures $\left(\nu_{x}^{u}\right)$ satisfying [H2] is referred to as a $\phi$-conformal systems of transversal measures ( $\phi$-cstm in abridged way). In 
[BM77, Hay94, Lep00], for different cases or with different proofs, it is proved that, up to a multiplicative constant, there exists a unique $\phi$-cstm satisfying in addition

$$
\forall x, \nu_{x}^{u}\left(W_{l o c}^{u}(x)\right)<+\infty .
$$

This system is the system $\left(\nu_{\phi, x}^{u}\right)$ and is referred to as the equilibrium $\phi$-conformal systems of transversal measures (e $\phi$-cstm in abridged way). Moreover if $\left(\nu_{x}^{u}\right)$ is a $\phi$ cstm and if for one $x, \nu_{x}^{u}\left(W_{l o c}^{u}(x)\right)$ is finite, then the systems $\left(\nu_{x}^{u}\right)$ is proportional to the unique $e \phi$-cstm. This is the so-called unique ergodicity of the horocycle foliation. This is related to the unique ergodicity of the horocycle flow in ergodic geometry for which there exists a large literature (see e.g. [Fur73, Dan78, Bur90, Cou01]).

Our second motivation for the paper was to understand where/why this unique ergodicity appears; more precisely, we want to understand where other natural candidates (see below) to be finite $\phi$-cstm effectively fail to be finite $\phi$-cstm.

In the other hand, K. Schmidt proved in [Sch77] that there exist other $\phi$-cstm. His proof does however not furnish explicit example. As a by-product of our study, our natural candidates to be finite $\phi$-cstm (but fail to be, due to the unique ergodicity) furnish such simple and explicit example of other $\phi$-cstm than the e $\phi$-cstm.

We emphasize that we are here dealing with two different dynamics. The dynamics of the holonomies, for which the $\phi$-cstm are relevant, and the dynamics of the shift $\sigma$ for which $\sigma$-invariant measures (like Gibbs states) are relevant.

Definition 1.1. Let $\left(\nu_{x}^{u}\right)$ be a $\phi$-cstm and $\mu$ be a $\sigma$-invariant measure. Let $\left(\mu_{x}^{u}\right)$ be the system of conditional measures with respect to the partition in local unstable leaves.

We say that the $\phi$-cstm is integrated by the measure $\mu$, and/or that the measure $\mu$ integrates the $\phi$-cstm if

(H1') for every $x, \nu_{x}^{u}$ is equivalent to $\mu_{x}^{u}$.

We are interested in finding $\phi$-cstm that are integrated by $\sigma$-invariant measures. For example, the unique $e \phi$-cstm is integrated by the unique Gibbs measure $\tau_{\phi}$.

Our candidates to be finite $\phi$-cstm are the following: considering a mixing subshift of finite type $\Sigma^{\prime} \subsetneq \Sigma$ and the associated Gibbs measure for $\left(\Sigma^{\prime}, \sigma\right)$, say $\tau_{\phi}^{\prime}$, there exists in $\Sigma^{\prime}$ a unique e $\phi$-cstm, say $\left(\nu_{\phi, x}^{\prime}\right)$. Clearly this systems $\left(\nu_{\phi, x}^{\prime}\right)$ is a candidate to be a "global" finite $\phi$-cstm. Why does this not hold?

Note that the measure $m$ obtained as the limit of $m_{\beta}$ is a barycenter of such $\nu_{\phi}^{\prime}$ (see [Lep05]). Our second result is

Theorem 2 Let $m$ be the limit measure $\lim _{\beta \rightarrow+\infty} m_{\beta}$. Let $\left(m_{x}^{u}\right)$ be its system of conditional measure with respect to the partition in local unstable leaves.

Then, there exists a $\phi$-cstm $\left(\nu_{R, x}^{u}\right)$ such that for every $x, m_{x}^{u}$ is equivalent to $\nu_{R, x}^{u}$. Moreover, there exists a $\sigma$-finite and $\sigma$-invariant measure $\tau$ which integrates $\left(\nu_{R, x}^{u}\right)$. The measure $\tau$ is not locally finite and not ergodic.

In particular, Theorem 2 gives explicit examples of $\phi$-cstm integrated by $\sigma$ invariant measures. Moreover, we have: 
Corollary 3 There are infinitely many non equivalent $\sigma$-finite measures which integrate $\phi$-conformal systems of transversal measures.

As far as we know, the question of infinite $\phi$-cstm has not been investigated a lot (at least for the compact case). For the non-compact case we mention works of Ledrappier and Sarig (see e.g. [LS07]).

As we said above, K. Schmidt proved in [Sch77], that, when a general dynamical system $(X, T)$ is uniquely ergodic, then it necessarily admits uncountable many $\sigma$-finite invariant measures. Existence of $\phi$-cstm different from the uniquee $\phi$-cstm results from this. However the proof in [Sch77] deeply uses a measurable correspondence between $\mathbb{Z}^{2}$-orbits and $\mathbb{Z}$-orbits; this orbital correspondence is not explicit at all.

In the same direction, L. Arnold gave a general condition for existence of invariant measure in the case of non-singular transformations in [Arn68]. We recall that a non-singular transformation is a dynamical system $(X, T)$ equipped with a measure of reference, $\mu$, such $\mu\left(T^{-1}(B)\right)=0$ if and only if $\mu(B)=0$. This result could be used for proving Theorem 2 and for the particular case $\phi \equiv 0$. For that, we should consider the system $\left(m_{x}^{u}\right)$ as the "measure of reference". This would however not work for general case, $\phi \not \equiv 0$.

Moreover, these two arguments could produce invariant measures for the holonomies, but it seems not clear that theses measures are integrable by $\sigma$-invariant measures.

Our construction is done when the system is a subshift of finite type. It also uses only one "rectangular hole" (see below). However, the same proof holds for Axiom-A diffeomorphisms or flows which are special flows. It should also work when we consider several "holes", but in that case, the dotted system would be more complicated.

Let us finish this introduction by mentioning a question related to our construction :

Question. Does it exist some probability measure, different from the equilibrium state $\mu_{\phi}$, invariant by the shift, which integrates a non-locally finite $\phi$-cstm?

Outline of the paper In the section 2 we precise the vocabulary used and recall some key points of [Lep00] for the construction of the measures $\check{\mu}_{\beta}$. In particular we introduce a new parameter, $Z=Z(\beta)$. We emphasize that our proofs use quite sophisticated estimates for the transfer operators. In this section we thus also recall some facts on the Transfer Operator Theory.

Section 3 is technical; we prove the convergence for some other objects (also depending on $\beta$ and/or $Z$ ). In section 4 we prove Theorem 1 using results from Section 3.

In section 5 we prove Theorem 2. We first construct the $\phi$-cstm defined by the system $\left(m_{x}^{u}\right)$, and then, we construct one $\sigma$-finite measure which integrates this system. We finish the section by proving that the construction leads to infinitely many non-equivalent measures (Corollary 3 ). 
2. Extra vocabulary, notations, recall on [Lep00]

\section{Extra vocabulary, notations, recall on [Lep00]}

In this section we give some more definitions and notations, make precise some vocabulary and some points in the theory of equilibrium states and transfer operator (see subsection 2.1). Then we define a subsystems and give properties of a oneparameter family of transfer operators. This parameter is denoted by $Z$. We explain the link between the two parameters $\beta$ and $Z$. Then we prove that $m_{\beta}$ converges as $\beta$ goes to $+\infty$.

\subsection{Vocabulary and notations}

For a given point $x=\left(x_{n}\right) \in \Sigma$, the past (resp. future) of the point denotes the backward (resp. forward) sequence $\left(x_{n}\right)_{n \leq 0}\left(\operatorname{resp} .\left(x_{n}\right)_{n \geq 0}\right)$. For $x$ and $y$ in $\Sigma$, when $x_{0}=y_{0}$, the point $z \stackrel{\text { def }}{=} \llbracket y, x \rrbracket$ is the point $\left(z_{n}\right)$ defined by $z_{n}=y_{n}$ if $n \leq 0$ and $z_{n}=x_{n}$ if $n \geq 0$.

Let $x=\left(x_{n}\right)$ and $y=\left(y_{n}\right)$ be two points in $\Sigma$. We set

$$
N(x, y):=\min n \geq 0, x_{n} \neq y_{n} \text { or } x_{-n} \neq y_{-n} .
$$

In $\Sigma$ the metric $d$ is given by $d(x, y)=\frac{1}{2^{N(x, y)}}$.

We recall that any $\alpha$-Hölder continuous function from $\Sigma$ to $\mathbb{R}$ is cohomologous to a $\frac{\alpha}{2}$-Hölder which is dependent only on the future (see [Bow75] Lemma 1.6). This assumption on $\phi$ was thus "free".

We also recall that the $\alpha$-norm is defined by

$$
\|\phi\|_{\alpha}=\sup _{x \neq y} \frac{|\phi(x)-\phi(y)|}{d^{\alpha}(x, y)} .
$$

If $x$ is in $\Sigma, C_{k, k+n}(x)$ denotes the cylinder $\left[i_{k}, \ldots, i_{k+n}\right]$ such that $x_{j}=i_{j}$ (for every $k \leq j \leq k+n)$. By extension, $C_{-\infty, n}(x)$ denotes the set of points $\left(y_{k}\right)$ such that $y_{k}=x_{k}$ for every $k \leq n$; similarly $C_{n,+\infty}(x)$ denotes the set of points $\left(y_{k}\right)$ such that $y_{k}=x_{k}$ for every $k \geq n$. By definition, the local unstable leaf $W_{l o c}^{u}(x)$ is $C_{-\infty, 0}(x)$, and the local stable leaf $W_{l o c}^{s}(x)$ is $C_{0,+\infty}(x)$.

\subsection{Thermodynamical formalism}

\subsubsection{Gibbs measures and equilibrium states}

The $e \phi$-cstm is equivalent to the system of conditional measures with respect to any measurable partition subordinate to the unstable leaves of the unique $\phi$-equilibrium state $\tau_{\phi}$. Recall that $\tau_{\phi}$, is the unique probability $\sigma$-invariant measure such that

$$
h_{\tau_{\phi}}(\sigma)+\int \phi d \tau_{\phi}=\sup _{\tau}\left\{h_{\tau}(\sigma)+\int \phi d \tau\right\} .
$$

The $e \phi-c s t m$ also satisfies

$$
\frac{d \nu_{\phi, x}^{u}}{d \sigma^{-1} \nu_{\phi, \sigma(x)}^{u}}=e^{\phi(x)-\mathcal{P}(\phi)},
$$


where $\mathcal{P}(\phi)=h_{\tau_{\phi}}(\sigma)+\int \phi d \tau_{\phi}$ is the pressure of $\phi$.

Remark 2. This is a usual tool in that theory to replace a condition on the holonomies like (3) by a condition on the shift like (5).

For a given $\sigma$-invariant measure $\lambda$, the $\phi$-pressure is the quantity $P_{\lambda}(\phi):=$ $h_{\lambda}(\sigma)+\int \phi d \lambda ; P_{\lambda}(\phi)$ will also be called the $\lambda$-pressure when there is no ambiguity on $\phi$.

\subsubsection{Transfer operator}

In a general way, for a two sided shift of finite type $\widetilde{\Sigma}, \widetilde{\Sigma}^{+}$will denote the forward one sided shift. A word is admissible for a shift $\widetilde{\Sigma}$ if it defines a non-empty cylinder in $\widetilde{\Sigma}$.

We now recall some element of the transfer operator theory. We refer to the book [Bal00] (Section 1.3 p. 28) for complete proof in general statements. In particular, the next facts are also valid for any uniformly expanding (or hyperbolic) dynamical system.

If $\psi: \widetilde{\Sigma}^{+} \rightarrow \mathbb{R}$ is Hölder continuous, the transfer operator or equivalently the Ruelle Perron Frobenius operator for $\left(\widetilde{\Sigma}^{+}, \sigma, \psi\right)$ is defined by

$$
\mathcal{L}(\mathcal{T})(x)=\sum_{\sigma(y)=x} e^{\psi(y)} \mathcal{T}(y)
$$

where $\mathcal{T}$ is a continuous function from $\widetilde{\Sigma}^{+}$to $\mathbb{R}$, and $x$ and $y$ are in $\widetilde{\Sigma}^{+}$. We call conformal measure (for $\psi$ ) the eigenmeasure for the adjoint operator of the transfer operator.

This measure $\nu_{\psi}$ is characterized by

$$
\mathcal{L}^{*}\left(\nu_{\psi}\right)=\left(\int \mathcal{L}\left(\mathbb{1}_{\widetilde{\Sigma}^{+}}\right) d \nu_{\psi}\right) \cdot \nu_{\psi},
$$

and it turns out that $\lambda:=\int \mathcal{L}\left(\mathbb{I}_{\widetilde{\Sigma}^{+}}\right) d \nu_{\psi}$ is the spectral radius for $\mathcal{L}$ and $\mathcal{L}^{*}$. Moreover, $\lambda$ is a dominating eigenvalue for $\mathcal{L}$, with eigenvector the positive Hölder continuous function

$$
H:=\lim _{n \rightarrow+\infty} \frac{1}{n} \sum_{k} \frac{1}{\lambda^{k}} \mathcal{L}^{k}\left(\mathbb{I}_{\widetilde{\Sigma}^{+}}\right) .
$$

Result 1. The function $H$ has the same Hölder regularity than $\psi$.

Result 2. The function $H$ is positive. Indeed, note that by construction it is non-negative. Now, the equality

$$
\lambda . H=\mathcal{L}(H),
$$

prove that if $H(x)=0$, then $H$ is null on a dense set, hence null everywhere by continuity. 
Result 3. The measure defined by $d \tau_{\psi}:=H d \nu_{\psi}$ is the unique equilibrium state for the potential $\psi$ in $\widetilde{\Sigma}^{+}$.

Remark 3. The system of measure $\left(\nu_{\phi, x}^{u}\right)$ from above is defined using this measure $\nu_{\psi}$ with $\psi=\phi$.

Result 4. There is a spectral decomposition for Hölder continuous functions:

$$
\mathcal{L}^{p}(\mathcal{T})(x)=\lambda^{p} \int \mathcal{T} d \nu_{\psi} H(x)+e^{p(\log \lambda-\varepsilon)} \mathcal{R}^{p}(\mathcal{T})(x),
$$

where $\left\|\mathcal{R}^{p}(\mathcal{T})\right\|_{\infty} \leq C\|\mathcal{T}\|_{\alpha}, \varepsilon$ and $C$ are positive real numbers which do not depend on $\mathcal{T}$ ( $\varepsilon$ is the spectral gap).

Result 5. The Doblin-Fortet inequality holds: there exist $0<a<1$ and $b>0$ and an integer $n_{0}$ such that for every Hölder function $\mathcal{T}$,

$$
\forall n \geq n_{0}, \quad\left\|\lambda^{-n} \mathcal{L}^{n}(\mathcal{T})\right\|_{\alpha} \leq a^{n} .\|\mathcal{T}\|_{\alpha}+b .\|\mathcal{T}\|_{\infty} .
$$

Now, if $\psi$ is defined on $\widetilde{\Sigma}$, the unique equilibrium state for $(\widetilde{\Sigma}, \sigma, \psi)$ is the natural extension in $\widetilde{\Sigma}$ of the unique equilibrium state for $\left(\widetilde{\Sigma}^{+}, \sigma, \psi^{+}\right)$, where $\psi^{+}$is the unique Hölder function cohomologous to $\psi$ and which does only depend on the future (constant on local stable leaves).

\subsection{Local thermodynamic formalism.}

In this section we define the local equilibrium states as in [Lep00].

Remember that the letter $R$ denotes one of the $(0,0)$-cylinders of $\Sigma$. It corresponds to the vertices $i_{R}$ in the alphabet of $\Sigma$. The letter $F$ will denote some local unstable leaf in $R$. Namely, $F$ denotes all the points in $R$ which have a same given past. The natural projection from $R$ onto $F$ is defined by $\pi_{F}(z)=\llbracket x, z \rrbracket$, where $x$ is any point in $F$.

For $x$ in $R, r(x)$ is the first return time in $R$ by iterations of $\sigma$ (if it exists) and $g$ is the first return map in $R$. We denote by $g_{F}$ the map $\pi_{F} \circ g$. Namely if $x=\left(x_{n}\right) \in F$ is given by

$$
\underbrace{\ldots, \xi_{-2}, \xi_{-1}}_{\text {past defined by } F}, \quad \stackrel{\downarrow}{i_{R}}, \underbrace{x_{1}, \ldots, x_{n-1}}_{\text {no } i_{R}}, i_{R}, x_{n+1}, \ldots,
$$

where $\downarrow$ indicates the initial position $x_{0}$, then $g(x)=\sigma^{n}(x)$ and is the infinite word

$$
\underbrace{\ldots, \xi_{-2}, \xi_{-1}}_{\text {past defined by } F}, i_{R}, \underbrace{x_{1}, \ldots, x_{n-1}}_{\text {no } i_{R}}, i_{R}^{\downarrow}, x_{n+1}, \ldots
$$

Then, $g_{F}(x)$ is the new infinite word

$$
\underbrace{\ldots, \xi_{-2}, \xi_{-1},}_{\text {past defined by } F}, \stackrel{\downarrow}{i_{R}, x_{n+1}, \ldots,}
$$


These maps $g$ and $g_{F}$ are not defined everywhere, because some points never return into $R$. Note however, that, due to the Markov property, the inverse branches of $g_{F}$ are well defined in the whole $F$ :

with the previous notations if $\underbrace{\ldots, \xi_{-2}, \xi_{-1},}_{\text {past defined by } F}, \stackrel{\downarrow}{i_{R}}, y_{1}, \ldots$ is a point $y$ in $F$, the point in $F \underbrace{\ldots, \xi_{-2}, \xi_{-1}}_{\text {past defined by } F}, \quad \stackrel{\downarrow}{{ }_{R}}, \underbrace{x_{1}, \ldots, x_{n-1}}_{\text {no } i_{R}}, i_{R}, y_{1}, \ldots$ is mapped by $g_{F}$ to $y$.

We can define the Ruelle-Perron-Frobenius operator for $g_{F}$ : for $x$ in $F$, we denote by $\operatorname{Pre}_{1}(x)$ the set of preimages of $x$ by $g_{F}$. For $y$ in $\operatorname{Pre}_{1}(x)$, we have $x=g_{F}(y):=\pi_{F} \circ \sigma^{r(y)}(y)$ and we set

$$
\Phi(y):=S_{r(y)}(\phi)(y)=\phi(y)+\ldots+\phi \circ \sigma^{r(y)-1}(y) .
$$

Then we set

$$
\mathcal{L}_{Z}(\mathcal{T})(x)=\sum_{y \in \operatorname{Pre}_{1}(x)} e^{\Phi(y)-r(y) \cdot Z} \mathcal{T}(y)
$$

where $\mathcal{T}: F \rightarrow \mathbb{R}$ is a continuous function, and $Z$ is a real parameter. Note that due to the Markov property, $\mathcal{L}_{Z}$ acts on continuous function defined on $F$; hence, the adjoint operator $\mathcal{L}_{Z}^{*}$ acts on the measures defined on $F$. This family of operators was studied in [Lep00]. There, we proved that there exists some critical $Z_{c}$, such that $\mathcal{L}_{Z}\left(\mathbb{1}_{F}\right)$ exists only for every $Z>Z_{c}$. Moreover, and always for $Z>Z_{c}, \mathcal{L}_{Z}$ admits a unique and single dominating eigenvalue $\lambda_{Z}$ in the set of $\alpha$-Hölder continuous functions. We also proved that the adjoint operator $\mathcal{L}_{Z}^{*}$ has $\lambda_{Z}$ for unique and single dominating eigenvalue.

Equilibrium state for $\left(F, g_{F}\right)$. Let us denote by $\nu_{Z}$ the unique probability measure on $F$ such that $\mathcal{L}_{Z}^{*}\left(\nu_{Z}\right)=\lambda_{Z} \cdot \nu_{Z}$. We denote by $H_{Z}$, the unique $\alpha$-Hölder continuous and positive function on $F$ satisfying $\mathcal{L}_{Z}\left(H_{Z}\right)=\lambda_{Z} \cdot H_{Z}$ and $\int H_{Z} d \nu_{Z}=$ 1. We also denote by $\mu_{Z}$ the measure $H_{Z} \cdot \nu_{Z}$. In [Lep00], we proved that $\mu_{Z}$ is a $g_{F^{-}}$ invariant probability measure. As we explained above at the end of Subsection 2.1, the measure $\mu_{Z}$ is an equilibrium state for the system $\left(F, g_{F}\right)$ and for the potential $\Phi-Z . r($.$) (here we use that \phi$ is constant along local stable leaves).

Equilibrium state for $(R, g)$. In [Lep00] we also proved that there exists a unique $g$-invariant probability measure with support in $R$ whose image by $\pi_{F}$ is $\mu_{Z}$. This measure is the measure $\widehat{\mu}_{Z}$ and is the natural extension of $\mu_{Z}$. It is the unique equilibrium state for the system $(R, g)$ and the potential $\Phi-\operatorname{Zr}($.$) .$

\subsection{Notations for measures}

One of the difficulties here comes from the large number of measures involved. We have thus adopted some fixed terminology and we shall explain it now. We also refer to Figure 1 page 13 to an overview of all the measures. 
The letter $\nu$ shall usually denote a conformal measure, that is an eigen-measure for some transfer operator as it is explained above. Note that formally, such a measure "leaves" in a one sided subshift of finite type.

The letter $\mu$ and in particular $\mu_{\square}$ shall designate a $g_{F}$-invariant measure on $F$. When the index in $\square$ is $Z$, then $\mu_{Z}$ is the unique equilibrium state for $g_{F}$ and for the potential $\Phi-Z . r($.$) . Later we shall change of parameter and use \beta=\beta(Z)$ instead of $Z$.

A measure $\widehat{\mu}$ and/or $\widehat{\mu}_{\square}$ is a $g$-invariant measure in $R$. It is usually seen as the natural extension of $\mu$ and/or $\mu_{\square}$. We shall later use the other parameter $\beta$ and the measure $\check{\mu}_{\beta}$ is the measure $\widehat{\mu}_{Z}$ with $\beta=\beta(Z)$, or equivalently $Z=Z(\beta)$.

A measure $\tau$ or $m$ shall usually refer to a $\sigma$-invariant measure in the whole subshift of finite type. The measure $m_{\square}$ is usually seen as the opened-out for $\widehat{\mu}_{\square}$, that is $\widehat{\mu}_{\square}$ is the restriction of $m_{\square}$ renormalized in $R$.

The equilibrium state for $\phi$ in $\Sigma$ is denoted by $\tau_{\phi}$. More generally, every $\lambda_{\square}$, where $\lambda$ is a measure and $\square$ contains $\phi$, denotes a measure related to the equilibrium state for $\phi$ (see e.g. $\left.\tau_{\phi}\right)$.

Measures of the form $\lambda_{\square, x}^{u}$ shall refer to measures on the local unstable leaf $W_{l o c}^{u}(x)$. If the "associated" measure $\lambda$ is $\sigma$-invariant (hence $\lambda_{\square}=\mu_{\square}$ or $m_{\square}$ ), then $\lambda_{\square, x}^{u}$ is the unique system of conditional measure. On the contrary if $\lambda=\nu$, then we refer to the invariance or absolutely continuity along for the holonomies along unstable leaves (see e.g. $\tau_{\phi, x}^{u}$ and $\nu_{\phi, x}^{u}$ in the introduction).

\subsection{More about $\mathcal{L}_{Z}$, value for $Z_{c}$, change of parameter}

We now recall some properties of the family of operators $\mathcal{L}_{Z}$. Remember that $\mathcal{L}_{Z}$ is the transfer operator associated to the map $g_{F}$. Hence, each $x$ in $F$ has infinitely many preimages by $g_{F}$, and one question is to check the convergence in the series which defines $\mathcal{L}_{Z}(\mathcal{T})(x)$.

Let us pick some $x$ in the unstable leaf $F \subset R$; we consider some $x^{\prime}$ in $\operatorname{Pre}_{1}(x)$, and some $y$ in $F$. The Markov property of $\sigma$ yields

$$
\pi_{F} \circ \sigma^{r\left(x^{\prime}\right)}\left(C_{0, r\left(x^{\prime}\right)}\left(x^{\prime}\right)\right)=F .
$$

It implies that $C_{0, r\left(x^{\prime}\right)}\left(x^{\prime}\right)$ contains a unique $y^{\prime} \in \operatorname{Pre}_{1}(y)$. Therefore, using the Hölder regularity of $\phi$, we get that there exists a constant $C_{\phi}$, which depends only on $\phi$, such that $\left|S_{r(x)}(\phi)\left(x^{\prime}\right)-S_{r(y)}(\phi)\left(y^{\prime}\right)\right| \leq C_{\phi}$. This holds for every pair of preimages. By induction, this holds for pair of preimages for the map $g_{F}^{n}$. Then, we get for every $n$ and for all $x$ and $y$ in $F$,

$$
e^{-C_{\phi}} \mathcal{L}_{Z}^{n}\left(\mathbb{I}_{F}\right)(x) \leq \mathcal{L}_{Z}^{n}\left(\mathbb{I}_{F}\right)(y) \leq e^{C_{\phi}} \mathcal{L}_{Z}^{n}\left(\mathbb{I}_{F}\right)(x) .
$$

Remember that $F$ is compact, and thus every continuous function on $F$ is bounded; hence (9) used for $n=1$ allows to prove that the series $\mathcal{L}_{Z}(\psi)(x)$ converges, for every point $x$ and for any continuous function $\psi$, as soon as $\mathcal{L}_{Z}\left(\mathbb{I}_{F}\right)(y)$ converges for one $y$. This defines $Z_{c}$ : for a given $y$ we write

$$
\mathcal{L}_{Z}\left(\mathbb{I}_{F}\right)(y)=\sum_{n=1}^{+\infty} a_{n}(y) e^{-n Z},
$$


which is a power series in $e^{-Z}$. Then we set $Z_{c}=\lim \sup _{n \rightarrow+\infty} \frac{1}{n} \log \left(a_{n}(y)\right)$. Hence for every $Z>Z_{c}$ the power series $\mathcal{L}_{Z}\left(\mathbb{I}_{F}\right)(y)$ converges.

In [CL05] (Subsection 3.3), it is proved that the critical value $Z_{c}$ is the pressure of the dotted system, with hole $R$, associated to $\phi$. Namely we consider in $\Sigma$ the sub-system $\Sigma_{R}$ of all the sequences $x=\left(x_{n}\right)_{n \in \mathbb{Z}}$ such that $x_{n}$ never equals $i_{R}$. Equivalently, $\Sigma_{R}$ is the set of points whose orbit never intersects the set $R$ (under the action of the shift $\sigma$ ). This subshift has for transition matrix the matrix $\mathcal{A}^{\prime}$ obtained from $\mathcal{A}$ by removing the line and the row corresponding to the vertices $i_{R}$. Up to the fact that this new system is mixing, it is proved in [CL05] that its $\phi$-pressure is the critical $Z_{c}$.

We now claim that the mixing hypothesis in [CL05] can be omitted. Indeed, any subshift of finite type can be decomposed in irreducible components, which satisfy the mixing property, but for some iteration of the map $\sigma$ (see [Ale76]). As we are considering first returns in $R$, note that the word defined by the cylinder $C_{0, r(x)-1}(x)$ contains no $i_{R}$ but at the first position. Hence, the word defined by $C_{1, r(x)-1}(x)$ is an admissible word for $\Sigma_{R}$ (if we assume that $r(x)>1$ ). By definition of the irreducible components, and by definition of $\Sigma_{R}$, it is an admissible word for a unique irreducible component of $\Sigma_{R}$.

Now, in a transitive subshift of finite type, the topological pressure associated to $\phi$ is the limit in $n$ of

$$
\frac{1}{n} \log \left(\sum e^{S_{n}(\phi)(\xi)}\right)
$$

where the sum is done over every words of length $n$ in the considered subshift, and we choose one point in the associated cylinder for each such word. It is however well-known, that the sum which defines the pressure, can be restricted to words whose initial position is a vertices in one fixed basic set. It is not necessary to consider all the words, but a sufficiently large number of them. To check this, just use Result 4 (page 8) with the appropriate $\mathcal{T}$.

If the system has several irreducible components, the pressure is defined as the maximum of all the pressure in the irreducible components. But as there are finitely many components, this is equivalent to consider the sum over all the words of length $n$. With these considerations, we point-out that the term $a_{n}(y)$ is exactly one such sum $\sum e^{S_{n}(\phi)(\xi)}$. Therefore $Z_{c}$ is the topological pressure of $\Sigma_{R}$ associated to $\phi$.

In [CL05] ( proof of Lemma 3.4), it was also proved that $\lambda_{Z} \rightarrow+\infty$ as $Z$ goes to $Z_{c}$. Moreover, the map $Z \mapsto \log \lambda_{Z}$ is a decreasing convex map on $] Z_{c},+\infty[$. By definition of $\nu_{Z}$ we have $\lambda_{Z}=\int \mathcal{L}_{Z}\left(\mathbb{I}_{F}\right) d \nu_{Z}$. Therefore (9) yields

$$
0 \leq \lambda_{Z} \leq e^{C_{\phi}} \mathcal{L}_{Z}\left(\mathbb{I}_{F}\right)(x) .
$$

The right hand side term is a power series in $e^{-Z}$, with valuation at least 1 . Therefore $\lambda_{Z}$ goes to 0 as $Z$ goes to $+\infty$.

Hence, the map $Z \mapsto \log \lambda_{Z}$ is a decreasing bijection from $] Z_{c},+\infty[$ onto $\mathbb{R}$. From now until the end of the paper, we set

$$
\beta=\beta(Z) \stackrel{\text { def }}{=} \log \lambda_{Z}
$$


As the map $Z \mapsto \log \lambda_{Z}$ is a bijection, we can consider the inverse map, $\beta \mapsto Z$ such that $\log \lambda_{Z}=\beta$. Then, $\beta \rightarrow+\infty$ is equivalent to $Z \rightarrow Z_{c}$. For the rest of the paper, we shall consider any of the two parameters, $\beta$ or $Z$, considering that when one is fixed, the other is also fixed.

\subsection{Convergence for $m_{\beta}$}

In [Lep00] it is proved that for every $Z>Z_{c}$ there exists a unique $\sigma$-invariant probability measure $m_{\beta(z)}^{\prime}$ such that

$$
\widehat{\mu}_{Z}=\frac{m_{\beta(Z)}^{\prime}(. \cap R)}{m_{\beta(Z)}^{\prime}(R)}
$$

Lemma 2.1. We have $m_{\beta}^{\prime}=m_{\beta}$, and for every $Z>Z_{c}$,

$$
h_{m_{\beta}}(\sigma)+\int \phi d m_{\beta}=Z+m_{\beta}(R) \beta \text {. }
$$

Proof. We have to prove that the measure $m_{\beta}^{\prime}=m_{Z}$ is the unique equilibrium state in $(\Sigma, \sigma)$ associated to $\phi-\beta(Z) . \mathbb{I}_{R}$.

Let us pick some $\sigma$-invariant probability measure $\tau$. We first assume that $\tau(R)>$ 0 . Then, we have,

$$
\begin{aligned}
h_{\tau}(\sigma)+\int \phi d \tau-Z & =\tau(R)\left(h_{\tau_{\mid R}}(g)+\int S_{r(.)}(\phi) d \tau_{\mid R}-Z . \int r(.) d \tau_{\mid R}\right) \\
& \leq \tau(R) \beta
\end{aligned}
$$

where $\tau_{\mid R}$ is the conditional measure $\tau(. \mid R)$, and where the last inequality is obtained by the variational principe. This gives

$$
h_{\tau}(\sigma)+\int \phi d \tau-\beta \cdot \int \mathbb{I}_{R} d \tau \leq Z
$$

with equality if and only if $\tau_{\mid R}=\widehat{\mu}_{Z}$ (i.e. $m_{Z}=m_{\beta}^{\prime}=\tau$ ).

If we assume that $\tau(R)=0$, then $\tau$ is a $\sigma$-invariant probability measure with support in $\Sigma_{R}$. Therefore it must satisfy

$$
h_{\tau}(\sigma)+\int \phi d \tau-\beta . \int \mathbb{I}_{R} d \tau=h_{\tau}(\sigma)+\int \phi d \tau \leq Z_{c}<Z .
$$

This proves that $m_{\beta}^{\prime}$ is the unique equilibrium state for $\phi-\beta . \mathbb{1}_{R}$, namely that we get $m_{\beta}^{\prime}=m_{\beta}$.

Remark 4. Following our notations, $\widehat{\mu}_{Z}=\check{\mu}_{\beta}$ with $\beta=\beta(Z)$. 


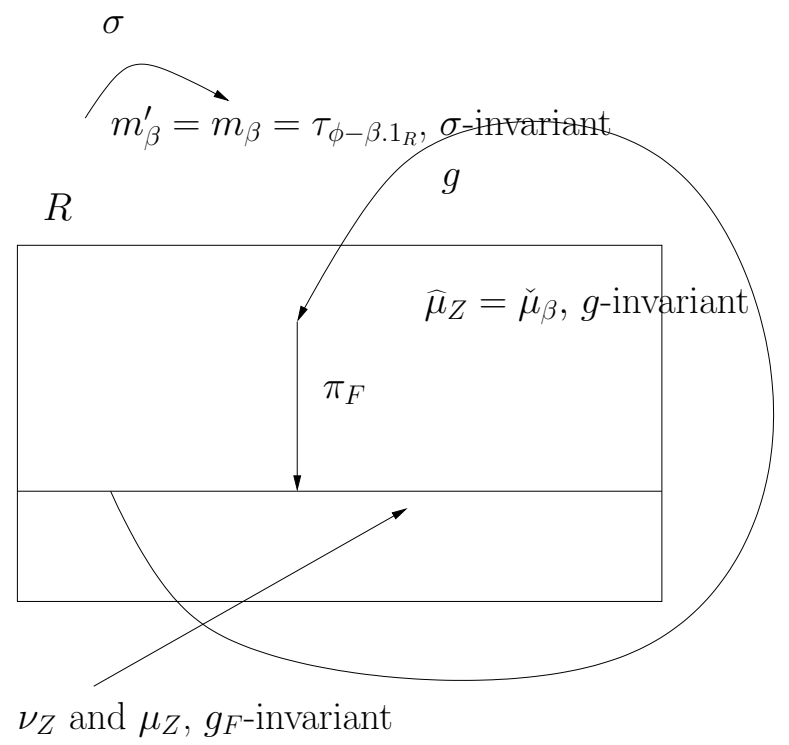

Figure 1: Local and global measures

As we said above, when $Z$ goes to $Z_{c}, \beta$ goes to $+\infty$; we thus use the theorem of convergence for equilibrium state a temperature zero (see [Lep05]) to get the convergence of $m_{\beta}$ to some limit measure $m$. This measure $m$ is maximizing for $-\mathbb{I}_{R}$, and we have seen that its supports is in $\Sigma_{R}$.

We now describe which irreducible components of the dotted systems $\Sigma_{R}$ have positive $m$-measure. It is well-known (see [CLT01]), that $m$ is a measure in $\Sigma_{R}$ with maximal $\phi$-pressure. Therefore $m$ weights only irreducible components of $\Sigma_{R}$ which have maximal $\phi$-pressure (which must be $Z_{c}$ ).

In [Lep05] we gave a way to identify the limit measure. For that, we introduced the notion of isolation rate between two irreducible components (with maximal $\phi$ pressure).

This isolation rate estimates the better way to join two irreducible components (and seems related to the Peierls barrier in [GLT10]). In the general case its calculation is relatively hard. In our case there is a unique way to join two irreducible components of the dotted system going through $i_{R}$. Then, every contribution for any link of that kind is the same (namely it is equal to $1=\mathbb{I}_{R}(x)$ ). Therefore, all the irreducible components have the same isolation rate and all the irreducible components of the dotted system $\Sigma_{R}$ with maximal $\phi$-pressure have positive $m$-measure.

Remark 5. If we make several holes, i.e., if $R$ is not a single $(0,0)$-cylinder, most of the previous results hold. However the irreducible components with maximal $\phi$-pressure would not necessarily all get the same isolation rate. This would make the rest of the proof more difficult. 


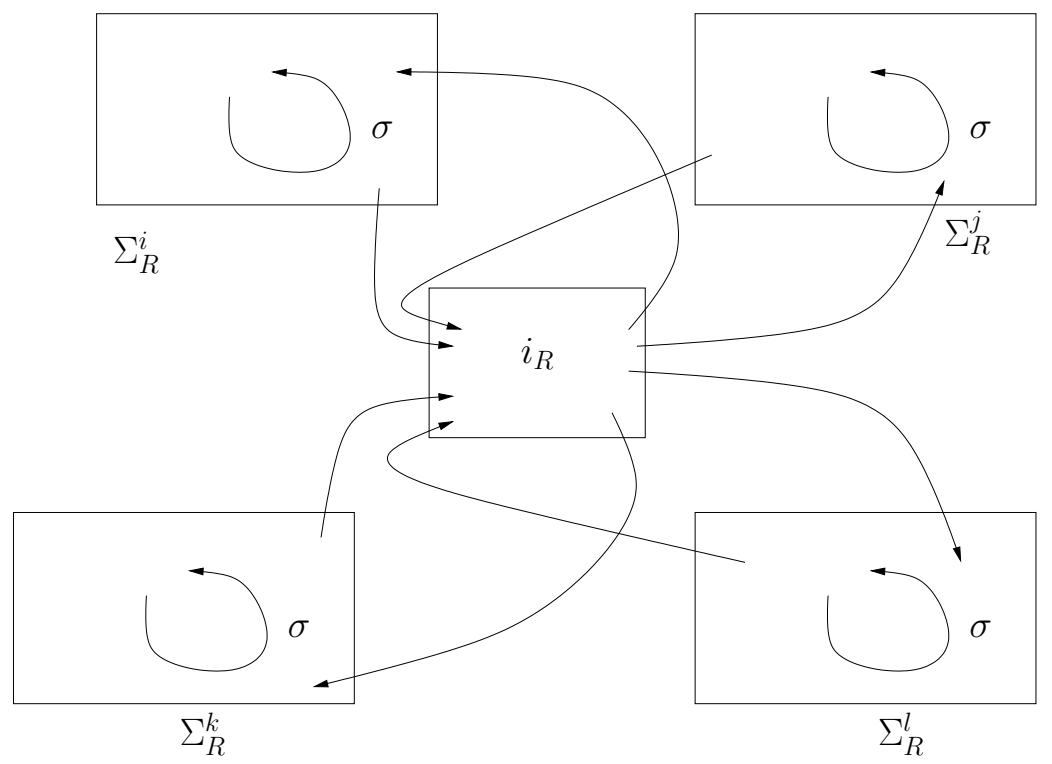

Figure 2: irreducible components, dynamics and transition costs

\section{Convergence for $H_{Z}, \nu_{Z}$ and $\mu_{Z}$}

The main result in this section is proposition 3.5 where we prove that $H_{Z}$ and $\nu_{Z}$ converge as $Z$ goes to $Z_{c}$. In Subsection 3.1 we state and prove one technical result which is the main tool to get the convergencies we mentioned above.

\subsection{Approximation of $\frac{1}{\lambda_{Z}} \mathcal{L}_{Z}$ as $Z$ goes to $Z_{c}$}

In this subsection we study the asymptotic for $\frac{1}{\lambda_{Z}} \mathcal{L}_{Z}$ when $Z$ goes to the critical value $Z_{c}$. In the first subsubsection we show how/why the dotted systems get greater importance for $Z$ close to $Z_{c}$. In the second subsubsection, we study the transfer operator for some relevant irreducible components of $\Sigma_{R}$. We also extend the sets of definition for these operators, and prove that some useful estimates are independent of $Z$. In the last subsubsection we give an explicit result for the estimation of the asymptotic.

\subsubsection{Influences of the dotted system when $Z$ goes to $Z_{c}$}

Lemma 3.1. Let us consider any accumulation point $\mu$ for the family $\left(\mu_{Z}\right)$ when $Z$ goes to $Z_{c}$. Then the set of point which return into $R$ by iterations of $\sigma$ has zero $\mu$-measure.

Proof. Let $x$ be in $F$ such that $r(x)<+\infty$. Let us set $n=r(x)$ and $K_{1}(x) \stackrel{\text { def }}{=}$ $C_{0, n}(x) \cap F$. Then we have $g_{F}\left(K_{1}(x)\right)=F$. Moreover (9) yields

$$
e^{-C_{\phi}} \cdot e^{S_{n}(\phi)(x)-Z . n-\beta} \leq \mu_{Z}\left(K_{1}(x)\right) \leq e^{C_{\phi}} \cdot e^{S_{n}(\phi)(x)-Z . n-\beta} .
$$


Therefore, when $Z$ goes to $Z_{c}, \beta$ goes to $+\infty$, and $\mu\left(K_{1}(x)\right)=0$.

Now the set of points which return into $R$ by iterations of $\sigma$ is the countable union of $K_{1}(x)$, when $x$ satisfies $r(x)<+\infty$. As any such set has zero $\mu$-measure, the union has zero $\mu$-measure. This finishes the proof.

This lemma explains the situation: the closer $Z$ is to $Z_{c}$, the more $\mathcal{L}_{Z}$ gives greater importance to orbits with long return time. But orbits with long return time in $R$ look almost like orbits in one of the irreducible component of $\Sigma_{R}$. Then, the closer $Z$ is to $Z_{c}$ the more $\frac{1}{\lambda_{Z}} \mathcal{L}_{Z}$ behaves like a transfer operator of $\Sigma_{R}^{+}$.

\subsubsection{Study of the dotted system and extensions of its thermody- namic tools}

In this subsubsection we define and study the transfer operator on $\Sigma_{R}$. We also extend the domains of definition of the thermodynamic notions.

We denote by $\Sigma_{R}^{l}, l=1, \ldots, P$ the irreducible components of $\Sigma_{R}$ with maximal $\phi$-pressure. For each one we denote by $\nu^{l}$ the conformal measure for $\phi$. The normalized eigenfunctions for the transfer operator are denoted by $\mathcal{H}_{l}, l=1, \ldots, P$. Remember that $\nu^{l}$ and $\mathcal{H}_{l}$ are in fact defined on the one sided shift $\Sigma_{R}^{l+}$.

- Gibbs measures and their extension.

For $x$ in $F$, with return time $n \leq+\infty$, we set

$$
\underbrace{\ldots, \xi_{-2}, \xi_{-1}}_{\text {past defined by } F}, \quad i_{R}^{\downarrow}, \underbrace{x_{1}, \ldots, x_{n-1}}_{\text {no } i_{R}}, i_{R}, \ldots
$$

The word $\left[x_{1}, \ldots, x_{n-1}\right]$ is an admissible word for a unique irreducible component of $\Sigma_{R}$. If this component is $\Sigma_{R}^{l}$, we then say that $x$ belongs to $F^{l}$. On $F^{l}$ we can define the measure $\nu^{l} \circ \sigma$ by

$$
\nu^{l} \circ \sigma(A)=\nu^{l}(\sigma(A))
$$

where we only consider the future of $\sigma(A)$ to compute $\nu^{l}(\sigma(A))$. This measure gives positive weight only for subsets of points in $F^{l}$ with infinite return time.

- Eigen-functions and their extensions.

Let us now pick some point $y=\left(y_{n}\right)_{n \geq 0}$ on the form

$$
\underbrace{y_{0}, \ldots, y_{n-1}}_{\text {admissible for } \Sigma_{R}^{l}}, i_{R}, \ldots
$$

Clearly $y$ does not belong to $\Sigma_{R}^{l+}$, and $\mathcal{H}_{l}(y)$ is a priori not defined. However, there is a canonical way to define it:

Remember that $\mathcal{H}_{l}$ satisfies $\mathcal{H}_{l}=\lim _{n \rightarrow+\infty} \frac{1}{n} \sum_{k} e^{-k Z_{c}} L_{l}^{k}\left(\mathbb{I}_{\Sigma_{R}^{l+}}\right)$ ( see (6) and note that the topological pressure is $Z_{c}$ ), where $L_{l}$ is the transfer operator in $\Sigma_{R}^{l+}$ for the potential $\phi$. But for $\xi$ in $\Sigma_{R}^{l+}, \sigma^{k}\left(\xi^{\prime}\right)=\xi$ simply means that we add in front of the one sided infinite word associated to $\xi$ an admissible word, with length $k$, for 
$\Sigma_{R}^{l+}$ (and it also has to satisfy some transition rules !). If $\xi$ starts with $y_{0}$, and if $\xi^{\prime}$ is

$$
\xi_{0}^{\prime}, \ldots, \xi_{k-1}^{\prime}, y_{0}, \xi_{1}, \ldots
$$

then the point $y^{\prime}:=\xi_{0}^{\prime}, \ldots, \xi_{k-1}^{\prime}, y_{0}, y_{1}, y_{2}, \ldots$, satisfies $\sigma^{k}\left(y^{\prime}\right)=y$ and it belongs to the same $(0, k)$-cylinder than $\xi^{\prime}$ (in $\left.\Sigma^{+}\right)$. Thus, we can set

$$
L_{l}^{k}\left(\mathbb{I}_{\Sigma_{R}^{l+}}\right)(y):=\sum_{y^{\prime}} e^{S_{k}(\phi)\left(y^{\prime}\right)},
$$

where the sum is done over all the possibilities for $y^{\prime}$, each one associated to one admissible word of length $k$ in $\Sigma_{R}^{l+}$. Then the definition of $\mathcal{H}_{l}(y)$ follows:

$$
\mathcal{H}_{l}(y)=\lim _{n \rightarrow+\infty} \frac{1}{n} \sum_{k} e^{-k Z_{c}} L_{l}^{k}\left(\mathbb{I}_{\Sigma_{R}^{l+}}\right)(y) .
$$

We let the reader check that this extension of $\mathcal{H}_{l}$ has the same Hölder regularity than $\mathcal{H}_{l}$ on $\Sigma_{R}^{l+}$. For convenience we now denote by $\widetilde{\Sigma}_{R}^{l+}$ the set of points $\xi_{0}, \xi_{1}, \ldots$, where $\left[\xi_{0}\right]$ is an admissible word for $\Sigma_{R}^{l+}$. Hence, we have extended the definition of $\mathcal{H}_{l}$ from $\Sigma_{R}^{l+}$ to $\widetilde{\Sigma}_{R}^{l+}$.

Lemma 3.2. The function $\mathcal{H}_{l}$ is positive on $\widetilde{\Sigma}_{R}^{l+}$.

Proof. Note that we still have $L_{l\left(\mathcal{H}_{l}\right)}=e^{Z_{c}} \mathcal{H}_{l}$. Moreover $\mathcal{H}_{l}$ is non-negative on $\widetilde{\Sigma}_{R}^{l+}$ and positive on $\Sigma_{R}^{l+}$ (see Result 2 page 7 ).

Let $y$ be in $\widetilde{\Sigma}_{R}^{l+} \backslash \Sigma_{R}^{l+}$. We assume that $\mathcal{H}_{l}(y)=0$. Let set $y^{\prime}:=\xi_{0}^{\prime}, \ldots, \xi_{k-1}^{\prime}, y_{0}, y_{1}, y_{2}, \ldots$, such that $\sigma^{k}\left(y^{\prime}\right)=y$ and $\left[\xi_{0}^{\prime}, \ldots, \xi_{k-1}^{\prime}\right]$ is admissible for $\Sigma_{R}^{l+}$. Then we get $\mathcal{H}_{l}\left(y^{\prime}\right)=0$. Doing $k \rightarrow+\infty$ and considering any accumulation point of the $y^{\prime}$ 's, we get a point in $\Sigma_{R}^{l+}$ where $\mathcal{H}_{l}$ vanishes. This is a contradiction with positivity of $\mathcal{H}_{l}$ in $\Sigma_{R}^{l+}$.

Now, let $x$ in $F$ be on the form

$$
\underbrace{\ldots, \xi_{-2}, \xi_{-1}}_{\text {past defined by } F}, i_{R}^{\downarrow}, x_{1}, \ldots
$$

and let $x^{\prime}$ in $F$ on the form

$$
\underbrace{\ldots, \xi_{-2}, \xi_{-1},}_{\text {past defined by } F}, i_{R}^{\downarrow}, \underbrace{x_{1}^{\prime}, \ldots, x_{n-1}^{\prime}}_{\text {no } i_{R}}, i_{R}, x_{1}, x_{2}, \ldots
$$

We have $g_{F}\left(x^{\prime}\right)=x$. Let us assume that $x_{1}^{\prime}, \ldots, x_{n-1}^{\prime}$ is an admissible word for $\Sigma_{R}^{l+}$. Following what we have done above, we can define $\mathcal{H}_{l}\left(\left[x_{n-1}^{\prime}, i_{R}, x_{1}, \ldots\right]\right)$. Then, we set

$$
\widetilde{\mathcal{H}}_{l}(x)=\sum \mathcal{H}_{l}\left(\left[x_{n-1}^{\prime}, i_{R}, x_{1}, \ldots\right]\right),
$$

where the sum is only done over all the different possible letters $x_{n-1}^{\prime}$ in the alphabet that defines $\Sigma_{R}^{l+}$. Namely if $x^{\prime \prime}$ in $F$ is on the form

$$
\underbrace{\ldots, \xi_{-2}, \xi_{-1}}_{\text {past defined by } F}, i_{R}^{\downarrow}, \underbrace{x_{1}^{\prime \prime}, \ldots, x_{m-1}^{\prime \prime}}_{\text {no } i_{R}}, i_{R}, x_{1}, x_{2}, \ldots,
$$


and $x_{m-1}^{\prime \prime}=x_{n-1}^{\prime}$, only one term $\mathcal{H}_{l}\left(\left[x_{n-1}^{\prime}, i_{R}, x_{1}, \ldots\right]\right)=\mathcal{H}_{l}\left(\left[x_{m-1}^{\prime \prime}, i_{R}, x_{1}, \ldots\right]\right)$ appears in the sum.

Remark 6. From Lemma 3.2 we get $\widetilde{\mathcal{H}}^{l}>0$.

- Transfer operators and their extension.

As we have just explained, the function $\mathcal{H}_{l}$ can be defined on a larger set than $\Sigma_{R}^{l+}$ The main reason for this, is that the transfer operator $L_{l}$ itself can be defined on continuous function from $\widetilde{\Sigma}_{R}^{l+}$ to $\mathbb{R}$. For any point $y$ in $\widetilde{\Sigma}_{R}^{l+}$, we only consider preimages in $\widetilde{\Sigma}_{R}^{l+}$ such that the added word (to the left of $y$ ) is admissible for $\Sigma_{R}^{l+}$ (just as above).

Lemma 3.3. The spectral radius of $L_{l}$ defined on the set $\mathcal{C}^{\alpha}\left(\widetilde{\Sigma}_{R}^{l+}, \mathbb{R}\right)$ of $\alpha$-Hölder function from $\widetilde{\Sigma}_{R}^{l+}$ to $\mathbb{R}$ is the same than the spectral radius of $L_{l}$ on $\mathcal{C}^{\alpha}\left(\Sigma_{R}^{l+}, \mathbb{R}\right)$. Moreover, we get

$$
L^{p}(\mathcal{T})(x)=e^{p . Z_{c}} \int \mathcal{T} d \nu^{l} \mathcal{H}_{l}(x)+e^{p\left(Z_{c}-\varepsilon\right)} \mathcal{R}_{l}^{p}(\mathcal{T})(x),
$$

where $\left\|\mathcal{R}_{l}^{p}(\mathcal{T})\right\|_{\infty} \leq C_{l}\|\mathcal{T}\|_{\alpha}$, $\varepsilon$ and $C_{l}$ are positive real numbers ( $\varepsilon$ is the spectral gap of the operator).

Proof. We just give the ideas of the proof. Indeed, these ideas are the same than in the proof of Proposition 3.5 (see below) and we believe it seems better to present a complete proof there.

The main ingredient to get the spectral decomposition is to use the IonescuTulcea and Marinescu theorem. There, the key point is to get the Dœblin-Fortet inequality (see Result 5 page 8 ). We claim, and let the reader check that this inequality effectively holds for $L_{l}$ on $\mathcal{C}^{\alpha}\left(\widetilde{\Sigma}_{R}^{l+}, \mathbb{R}\right.$ ) (with the same proof than for $L_{l}$ on $\left.\mathcal{C}^{\alpha}\left(\Sigma_{R}^{l+}, \mathbb{R}\right)\right)$.

Now, the second point in the proof is to check that the spectral radius is also the eigenvalue for the adjoint operator (acting on measures). But the unique eigenmeasure is $\nu^{l}$, considering the adjoint operator either acting on measures defined on $\widetilde{\Sigma}_{R}^{l+}$ or on $\Sigma_{R}^{l+}$. This shows that the two operators have the same spectral radius, $e^{Z_{c}}$, and that the decomposition holds for Hölder continuous functions.

\subsubsection{Asymptotic for $\frac{1}{\lambda_{Z}} \mathcal{L}_{Z}$}

Proposition 3.4. With the previous notations, there exists a positive $\varepsilon$ such that for every $\mathcal{T}: F \rightarrow \mathbb{R} \alpha$-Hölder continuous and for every $x$ in $F$,

$$
\begin{aligned}
\frac{1}{\lambda_{Z}} \mathcal{L}_{Z}(\mathcal{T})(x)= & \frac{e^{-\beta}}{1-e^{Z_{c}-Z}} \sum_{l=1}^{P}\left(\int_{F^{l}} \mathcal{T}(\xi) e^{\phi(\xi)-Z} d \nu^{l} \circ \sigma(\xi)\right) \times \widetilde{\mathcal{H}}_{l}(x) \\
& +\frac{e^{-\beta}}{1-e^{Z_{c}-Z-\varepsilon}} \cdot \mathcal{O}\left(\|\mathcal{T}\|_{\alpha}\right)
\end{aligned}
$$


Proof. Let us pick some $x$ in $F$ and some $\mathcal{T}$. We have

$$
\frac{1}{\lambda_{Z}} \mathcal{L}_{Z}(\mathcal{T})(x)=e^{-\beta} \sum_{y \in \operatorname{Pre}_{1(x)}} e^{\Phi(y)-Z . r(y)} \mathcal{T}(y) .
$$

For such $y$ in $\operatorname{Pre}_{1}(x)$ we set

$$
\underbrace{\ldots, \xi_{-2}, \xi_{-1}}_{\text {past defined by } F}, \quad i_{R}^{\downarrow}, \underbrace{y_{1}, \ldots, y_{n-1}}_{\text {no } i_{R}}, i_{R}, x_{1}, x_{2}, \ldots
$$

We focus our attention on such $y$ such that $y_{1}, \ldots, y_{n-1}$ is admissible for some $\Sigma_{R}^{l+}$; during the computation, it will appear why other points give a negligible contribution.

In the rest of the proof we say that such a preimage visits the irreducible component $\Sigma_{R}^{l}$.

Now, remember that $\phi$ does only depend on the future. Then, considering all the preimages by $g_{F}$ of the point $x$ which visit $\Sigma_{R}^{l}$, we pack away the preimages in function of the first letter $y_{1}$ and the last letter before $i_{R}, y_{r(y)-1}$. The sum over these preimages satisfies

$$
\begin{gathered}
\sum_{\substack{y \text { visiting } \\
\Sigma_{R}^{l}}} e^{\Phi(y)-Z r(y)} \mathcal{T}(y)=\sum_{\substack{\text { possible } \\
y_{1}}} \sum_{\substack{\text { possible } \\
x_{-1}}} e^{\phi(y)} \mathcal{T}(y) e^{S_{r(y)-2}(\phi)(\sigma(y))} e^{\phi\left(\left[x_{-1}, i_{R}, x_{1}, \ldots\right]\right)} e^{-Z r(y)} \\
=\sum_{\substack{\text { possible } \\
x_{-1}}} \sum_{p=0}^{+\infty} e^{-(p+1) Z} L_{l}^{p}\left(e^{\phi \circ \sigma^{-1}} \mathcal{T} \circ \sigma^{-1}\right)\left(\left[x_{-1}, i_{R}, x_{1}, \ldots\right]\right),
\end{gathered}
$$

where $\sigma^{-1}(\xi)$ means the concatenation in $\widetilde{\Sigma}_{R}^{l+}, \llbracket i_{R}, \xi \rrbracket$. Now (13) yields

$$
\begin{aligned}
L^{p}\left(e^{\phi \circ \sigma^{-1}} \mathcal{T} \circ \sigma^{-1}\right)\left(\left[x_{-1}, i_{R}, x_{1}, \ldots\right]\right)= & e^{p . Z_{c}}\left(\int e^{\phi \circ \sigma^{-1}} \mathcal{T} \circ \sigma^{-1} d \nu^{l}\right) \times \mathcal{H}_{l}\left(\left[x_{-1}, i_{R}, x_{1}, \ldots\right]\right) \\
& +e^{p\left(Z_{c}-\varepsilon\right)} \mathcal{R}_{l}^{p}\left(e^{\phi \circ \sigma^{-1}} \mathcal{T} \circ \sigma^{-1}\right)\left(\left[x_{-1}, i_{R}, x_{1}, \ldots\right]\right) .(16)
\end{aligned}
$$

Therefore, doing the sum over all the integers $p$ and all the possible $x_{-1}$ in (15), (11), (12) and (16) yield

$$
\begin{aligned}
\sum_{\substack{y \text { visiting } \\
\Sigma_{R}^{l}}} e^{\Phi(y)-Z r(y)} \mathcal{T}(y)= & \frac{1}{1-e^{Z-Z_{c}}}\left(\int_{F^{l}} e^{\phi-Z} \mathcal{T} d \nu^{l} \circ \sigma\right) \times \widetilde{\mathcal{H}}_{l}(x) \\
& +\frac{1}{1-e^{Z-Z_{c}-\varepsilon}} e^{\|\phi\|_{\infty}-Z} \mathcal{O}\left(\|\mathcal{T}\|_{\alpha}\right) .
\end{aligned}
$$

Here appears why preimages visiting other components than the $\Sigma_{R}^{l}$ give a negligible contribution. These components have a $\phi$-pressure strictly smaller than $Z_{c}$. Hence 
the spectral radius for their transfer operators are strictly smaller than $e^{Z_{c}}$. Doing the same computation than above, the first term in the right hand size of (17) should be replaced by $\frac{1}{1-e^{Z-Z_{c}-\varepsilon}}$, where $e^{Z_{c}-\varepsilon}$ is the spectral radius of the operator for the visited component.

Note that a key point here is that $\Sigma_{R}$ has finitely many irreducible components: this allows to find a uniform positive $\varepsilon$.

This finishes the proof of the proposition.

\subsection{Convergencies}

In this subsection, we are going to use Proposition 3.4 to prove the expected convergencies.

Proposition 3.5. The function $H_{Z}$, the measure $\nu_{Z}$ and the measure $\mu_{Z}$ converge as $Z$ goes to $Z_{c}$

Proof. The main idea is to prove that the quantities we are studying have a unique accumulation point when $Z$ goes to $Z_{c}$, or equivalently, as $\beta$ goes to $+\infty$. During the proof the quantity $\frac{e^{-\beta}}{1-e^{Z-Z_{c}}}$ and its possible accumulation points are going to play a key role.

- First step: The $H_{Z}$ 's are equi-continuous.

Remember that $H_{Z}$ is defined by

$$
H_{Z}=\lim _{n \rightarrow+\infty} \frac{1}{n} \sum_{k<n} e^{-k \beta} \mathcal{L}_{Z}^{k}\left(\mathbb{I}_{F}\right)=\lim _{n \rightarrow+\infty} \frac{1}{n} \sum_{n_{0} \leq k<n} e^{-k \beta} \mathcal{L}_{Z}^{k}\left(\mathbb{I}_{F}\right) .
$$

The equi-continuity shall follow from the Dœblin-Fortet inequality.

Let $\mathcal{T}$ be any Hölder function on $F$. Result 5 (see page 8 )says that we get a bound of the form

$$
\forall n \geq n_{0}, \quad\left\|e^{-n \beta} \mathcal{L}_{Z}^{n}(\mathcal{T})\right\|_{\alpha} \leq a^{n} .\|\mathcal{T}\|_{\alpha}+b .\|\mathcal{T}\|_{\infty} .
$$

The important point is that $C_{\phi}, n_{0}, a$ and $b$ do not depend on $Z$ but only on $\phi$.

Indeed, we already saw that $C_{\phi}$ is a bound for the variation of $S_{n}(\phi)$ on all the $(0, n)$-cylinders. The integer $n_{0}$ and the constants $a$ and $b$ are obtained computing the $\alpha$-norm of $e^{-n \beta} \mathcal{L}_{Z}^{n}(\mathcal{T})$. We get:

$$
\begin{aligned}
\left|e^{-n \beta} \mathcal{L}_{Z}^{n}(\mathcal{T})(x)-e^{-n \beta} \mathcal{L}_{Z}^{n}(\mathcal{T})(y)\right| \leq & \frac{\|\mathcal{T}\|_{\alpha}}{e^{n \beta}} \frac{d^{\alpha}(x, y)}{2^{n}} \mathcal{L}_{Z}^{n}\left(\mathbb{I}_{F}\right)(x) \\
& +\frac{\|\mathcal{T}\|_{\infty}}{e^{n \beta}}\left|e^{C_{\phi} \frac{d^{\alpha}(x, y)}{2^{n}}}-1\right| \mathcal{L}_{Z}^{n}\left(\mathbb{I}_{F}\right)(x) .
\end{aligned}
$$

Then (9) yields

$$
\left\|e^{-n \beta} \mathcal{L}_{Z}(\mathcal{T})\right\|_{\alpha} \leq 2^{-n} e^{C_{\phi}}\|\mathcal{T}\|_{\alpha}+C . C_{\phi} 2^{-n}\|\mathcal{T}\|_{\infty} .
$$


We pick $n_{0}$ large enough that $a:=2^{-n_{0}} e^{C_{\phi}}<1$. Clearly neither $a$, nor $n_{0}$ depend on $Z$. Then we get $b$ using the standard argument of the euclidean division of $n$ by $n_{0}$ when we compute $\left\|e^{-n \beta} \mathcal{L}_{Z}(\mathcal{T})\right\|_{\alpha}$ for large $n$.

Therefore, using the Doblin-Fortet inequalities, the fact that the constants do not depend on $Z$ and the definition of $H_{Z}$ we get that all the functions $H_{Z}$ are equi-continuous.

Using the Ascoli theorem, we can get some convergent subsequence from any subfamily. Moreover, the set of function $\left\{H_{Z}, Z \geq Z_{c}\right\}$ is bounded from above for the $\alpha$-norm (by $b$ ). Then, even if the convergence occurs for the $\infty$-norm, the limit is $\alpha$-Hölder with $\alpha$-norm lower than $b$.

- Second step: $\frac{e^{-\beta}}{1-e^{Z-Z_{c}}}$ and $\nu_{Z}$ converge. We first pick a subfamily of $Z$ such that $\frac{e^{-\beta}}{1-e^{Z_{c}-S}}$ converges to $\mathcal{A}$ when $Z$ goes to $Z_{c}$ and $Z$ belongs to the subfamily. For convenience we write $\lim _{Z \downarrow Z_{c}}$, thinking limit for the selected subfamily.

Again, $\left\{\nu_{Z}\right\}$ is a pre-compact family, and we can extract some convergent subfamily from the previous subfamily. Let us consider some accumulation point for $\nu_{Z}$, respecting the previous convergence.

The main idea to prove the uniqueness of $\nu$ is to prove that $\int \mathcal{T} d \nu$ is uniquely determined for any Hölder $\mathcal{T}$.

Let us pick some $\mathcal{T}$.

By Proposition 3.4 we have for every $x$,

$$
\begin{aligned}
\frac{1}{\lambda_{Z}} \mathcal{L}_{Z}(\mathcal{T})(x)= & \frac{e^{-\beta}}{1-e^{Z_{c}-Z}} \sum_{l=1}^{P}\left(\int_{F^{l}} \mathcal{T} e^{\phi-Z} d \nu^{l} \circ \sigma\right) \times \widetilde{\mathcal{H}}_{l}(x) \\
& +\frac{e^{-\beta}}{1-e^{Z_{c}-Z-\varepsilon}} \cdot \mathcal{O}\left(\|\mathcal{T}\|_{\alpha}\right)
\end{aligned}
$$

Note that $\lim _{Z \downarrow Z_{c}} \frac{e^{-\beta}}{1-e^{Z_{c}-Z-\varepsilon}}=0$ because $\varepsilon$ is positive and $\beta$ goes to $+\infty$.

In the other hand we get

$$
\nu_{Z}(\mathcal{T})=\frac{1}{\lambda_{Z}} \mathcal{L}_{Z}^{*}\left(\nu_{Z}\right)(\mathcal{T})=\int \frac{1}{\lambda_{Z}} \mathcal{L}_{Z}(\mathcal{T}) d \nu_{Z}
$$

Now, (18) yields

$$
\int \mathcal{T} d \nu=\mathcal{A} \cdot \sum_{l}\left(\int_{F^{l}} \mathcal{T} e^{\phi-Z_{c}} d \nu^{l} \circ \sigma\right) \int \widetilde{\mathcal{H}}_{l} d \nu .
$$

We now use (19) for $\mathcal{T}=\mathbb{I}_{F}$; remember that for every $Z$ we have $\nu_{Z}(F)=1$. Therefore we get

$$
1=\mathcal{A} \sum_{l}\left(\int_{F^{l}} e^{\phi-Z_{c}} d \nu^{l} \circ \sigma\right) \int \widetilde{\mathcal{H}}_{l} d \nu
$$

Note that all the numbers $\int \widetilde{\mathcal{H}}_{l} d \nu$ are positive (follows from Remark 6 ). Therefore (20) implies that $\mathcal{A}$ belongs to $] 0,+\infty[$. 
Using (19) with $\mathcal{T}=\widetilde{\mathcal{H}}_{j}$ we get

$$
\frac{1}{\mathcal{A}} \int \widetilde{\mathcal{H}}_{j} d \nu=\sum_{l}\left(\int_{F^{l}} \widetilde{\mathcal{H}}_{j} e^{\phi-Z_{c}} d \nu^{l} \circ \sigma\right) \int \widetilde{\mathcal{H}}_{l} d \nu
$$

This means that the vector $\left(\begin{array}{c}\int \widetilde{\mathcal{H}}_{1} d \nu \\ \vdots \\ \int \widetilde{\mathcal{H}}_{P} d \nu\end{array}\right)$ is a positive eigenvector for the matrix $\mathbb{M}$ with entries $\left(\int_{F^{l}} \widetilde{\mathcal{H}}_{j} e^{\phi-Z_{c}} d \nu^{l} \circ \sigma\right)_{j, l}$. This matrix has all its entries positive, and we can use the Perron-Frobenius theorem. The matrix admits a unique (up to a multiplicative factor) eigenvector with all positive entries and the associated eigenvalue is the spectral radius of the matrix $\mathbb{M}$. It equals $\frac{1}{\mathcal{A}}$.

Note that the considered matrix does not depend on the accumulations points we considered. This proves that $\mathcal{A}$ is uniquely determined, hence $\frac{e^{-\beta}}{1-e^{Z-Z_{c}}}$ converges as $Z$ goes to $Z_{c}$. Now, all the $\int \widetilde{\mathcal{H}}_{j} d \nu$ are uniquely determined up to a multiplicative constant, and (20) fixes their respective value. They are thus uniquely determined, and(19) implies that $\nu$ is uniquely determined; hence $\nu_{Z}$ converges as $Z$ goes to $Z_{c}$.

- Step three: $H_{Z}$ converges. Our strategy is (as before) to prove that the family $\left\{H_{Z}\right\}$ admits a unique accumulation point, hence its convergence. Let us consider some accumulation point $H$ for $H_{Z}$ (for the norm of uniform convergence).

Then

$$
H=\lim _{Z \downarrow Z_{c}} H_{Z}=\lim _{Z \downarrow Z_{c}} \frac{1}{\lambda_{Z}} \mathcal{L}_{Z}\left(H_{Z}\right)
$$

and (18) yields

$$
H=\mathcal{A} \cdot \sum_{l=1}^{P}\left(\int_{F^{l}} H e^{\phi-Z_{c}} d \nu^{l} \circ \sigma\right) \widetilde{\mathcal{H}}_{l} .
$$

This yields for every $x$ in $F$

$$
\begin{aligned}
H(x) & =\mathcal{A} \cdot \sum_{l}\left(\int_{F^{l}} H e^{\phi-Z_{c}} d \nu^{l} \circ \sigma\right) \widetilde{\mathcal{H}}_{l}(x) \\
& =\mathcal{A}^{2} \sum_{j, l}\left(\int_{F^{j}} H e^{\phi-Z_{c}} d \nu^{j} \circ \sigma\right)\left(\int_{F^{l}} \widetilde{\mathcal{H}}_{j} e^{\phi-Z_{c}} d \nu^{l} \circ \sigma\right) \widetilde{\mathcal{H}}_{l}(x) .
\end{aligned}
$$

where we obtain the second equation by replacing $H$ in the term $\int H e^{\phi-Z_{c}} d \nu^{l} \circ \sigma$ of the first equation by the right hand side term of (22) .

Note that we can iterate this process, inserting (22) in (23) and so on. Therefore we get a family of equation on the form

$$
\left(\vec{a} \mid \mathbb{A}^{n} \vec{b}(x)\right)=\frac{H(x)}{\mathcal{A}}, n \geq 0, x \in F
$$


where $\mathbb{A}$ is the matrix $\mathcal{A} \mathbb{M}$ with positive entries $\left(\mathcal{A} \int_{F^{l}} \widetilde{\mathcal{H}}_{j} e^{\phi-Z_{c}} d \nu^{l} \circ \sigma\right)_{j l}$, and

$$
\begin{gathered}
\vec{a}=\left(\int_{F^{1}} H e^{\phi-Z_{c}} d \nu^{1} \circ \sigma, \ldots, \int_{F^{P}} H e^{\phi-Z_{c}} d \nu^{P} \circ \sigma\right), \\
\vec{b}(x)=\left(\begin{array}{c}
\widetilde{\mathcal{H}}_{1}(x) \\
\vdots \\
\widetilde{\mathcal{H}}_{P}(x)
\end{array}\right) .
\end{gathered}
$$

The matrix $\mathbb{A}^{*}$ has positive entries and spectral radius 1 . Using Perron-Frobenius theorem, we get that $\vec{a} \cdot \mathbb{A}^{n}$ converges as $n$ goes to $+\infty$ to $(\vec{a} \mid \vec{u})$, where $\vec{u}$ is the unique normalized vector with positive entries such that $\vec{u} . \mathbb{A}=\vec{u}$. This proves that $H$ is uniquely determined. Therefore $H_{Z}$ converges.

As the convergence occurs uniformly, we directly get convergence for $\mu_{Z}$; indeed $d \mu_{Z}=H_{Z} d \nu_{Z}$. This finishes the proof of the proposition.

Remark 7. Note that $H$ is a positive function on $F$.

Relations (19) and (20) yield an important result for the rest of the proof:

Proposition 3.6. There exist $P$ positive constants $a_{l}, l=1, \ldots, P$ such that for every $l$ and for every $x$ in $F^{l}$,

$$
\frac{d \nu}{d \nu^{l} \circ \sigma}=a_{l} \cdot e^{\phi(x)-Z_{c}}
$$

\section{Convergence for $\widehat{\mu}_{Z}$ and asymptotic of the limit measure}

In the section, we prove that $\widehat{\mu}_{Z}$ converges as $Z$ goes to $Z_{c}$. We also prove that the limit $\widehat{\mu}$ satisfies the required properties. To prove convergence for $\widehat{\mu}_{Z}$ it is equivalent to prove convergence for $\widehat{\mu}_{Z}\left(C_{-n, m}(x)\right)$ for any non-negative integers $n, m$, and for any $x$ in $R$. Using the disintegration of the measure along the stable leaves, we get

$$
\widehat{\mu}_{Z}\left(C_{-n, m}(x)\right)=\int \widehat{\mu}_{Z, y}^{s}\left(C_{-n, 0}(x)\right) \mathbb{I}_{C_{0, m}(x)}(y) d \mu_{Z}(y),
$$

where $\widehat{\mu}_{Z, y}^{s}$ is the disintegrated measure on the fiber $C_{0,+\infty}(y)$. We already have the convergence for $\mu_{Z}$; we only need the uniform convergence for the map $y \mapsto$ $\widehat{\mu}_{Z, y}^{s}\left(C_{-n, 0}(x)\right)$. This will follow from the next two lemmas.

Let us pick some $y$. We use proposition 7.1 in [Lep00] to get

$$
\begin{aligned}
\widehat{\mu}_{Z, y}^{s}\left(C_{-n, 0}(x)\right) & =\sum_{y^{\prime} \in \operatorname{Pre}_{1}(y)} \widehat{\mu}_{Z, y}^{s}\left(g\left(\eta^{s}\left(y^{\prime}\right)\right)\right) \mathbb{I}_{C_{-n, 0}(x)}\left(g\left(y^{\prime}\right)\right) \\
& =\sum_{y^{\prime} \in g^{-1}\left(C_{-n, 0}(x)\right)} \frac{H_{Z}\left(y^{\prime}\right)}{H_{Z}(y)} e^{\Phi\left(y^{\prime}\right)-Z \cdot r\left(y^{\prime}\right)-\beta} .
\end{aligned}
$$

Remember that $g$ is the map $\sigma^{r(.)}($.$) , and thus, the last sum is taken over the y^{\prime}$ in $\operatorname{Pre}_{1}(y)$ such that $g\left(y^{\prime}\right) \in C_{-n, 0}(x)$. 
Lemma 4.1. If there exists $y^{\prime}$ in $\operatorname{Pre}_{1}(y)$ such that $r\left(y^{\prime}\right)=n$, then $\widehat{\mu}_{Z, \xi}^{s}\left(C_{-n, 0}(x)\right)$ goes uniformly (in $\xi$ ) to 0 as $Z$ goes to $Z_{c}$.

Proof. Remember we defined the 1-sets in the proof of Lemma 3.1. With our notations, $K_{1}\left(y^{\prime}\right)=F \cap C_{0, n}\left(y^{\prime}\right)$. Moreover,

$$
g\left(C_{0, n}\left(y^{\prime}\right)\right)=\sigma^{n}\left(C_{0, n}\left(y^{\prime}\right)\right)=C_{-n, 0}(x) .
$$

Therefore, (25) yields $\widehat{\mu}_{Z, \xi}^{s}\left(C_{-n, 0}(x)\right)=\frac{H_{Z}\left(\xi^{\prime}\right)}{H_{Z}(\xi)} e^{\Phi\left(\xi^{\prime}\right)-Z_{c} n-\beta}$, where $\xi^{\prime}$ is the unique element in $\operatorname{Pre}_{1}(\xi) \cap K_{1}\left(y^{\prime}\right)$. Now, when $Z$ goes to $Z_{c}, \beta$ goes to $+\infty, n$ is fixed, and thus $\widehat{\mu}_{Z, \xi}^{s}\left(C_{-n, 0}(x)\right)$ goes uniformly to 0 .

Let us set $C_{-n, 0}(x)=\left[x_{-n}, x_{-n+1}, \ldots, x_{-1}, i_{R}\right]$. Lemma 4.1 implies that if one $x_{-j}=i_{R}$ with $0<j \leq n$, then $\widehat{\mu}_{Z, \xi}^{s}\left(C_{-n, 0}(x)\right)$ goes uniformly (in $\xi$ ) to 0 as $Z$ goes to $Z_{c}$. We now consider the case where no $x_{-j}$ equals $i_{R}$ (except $x_{0}$ ). Note that $\left[x_{-n}, \ldots, x_{-1}\right]$ is an admissible word for an unique irreducible component of $\Sigma_{R}$

Lemma 4.2. There exists some positive $\varepsilon$ such that, if $\left[x_{-n}, \ldots, x_{-1}\right]$ is an admissible word for $\Sigma_{R}^{l}$, then

$$
\begin{aligned}
\widehat{\mu}_{Z, y}^{s}\left(C_{-n, 0}(x)\right)= & \frac{1}{H_{Z}(y)} e^{S_{n}(\phi)\left(\sigma^{-n}(y)\right)-n Z}\left(\mathcal{O}\left(\frac{e^{-\beta}}{1-e^{Z_{c}-Z-\varepsilon}}\right)+\right. \\
& \left.\left(\int_{F^{l}} H_{Z} e^{\phi-Z} d \nu^{l} \circ \sigma\right) \mathcal{H}_{l}\left(\sigma^{-n}(y)\right) \frac{e^{-\beta}}{1-e^{Z_{c}-Z}}\right),
\end{aligned}
$$

where $\sigma^{-n}(y)$ denotes $\left[x_{-n}, x_{-n+1}, \ldots, x_{-1}, i_{R}, y_{1}, y_{2}, \ldots\right]$.

If $\left[x_{-n}, \ldots, x_{-1}\right]$ is not an admissible word for any $\Sigma_{R}^{l}$, then

$$
\widehat{\mu}_{Z, y}^{s}\left(C_{-n, 0}(x)\right)=\mathcal{O}\left(\frac{e^{-\beta}}{1-e^{Z_{c}-Z-\varepsilon}}\right)
$$

Proof. We copy the proof of Proposition 3.4. We first assume that $\left[x_{-n}, \ldots, x_{-1}\right]$ is an admissible word for $\Sigma_{R}^{l}$. The other case will follow from the same computation. Equation (25) yields

$$
\begin{aligned}
\sum_{y^{\prime} \in g^{-1}\left(C_{-n, 0}(x)\right)} \frac{H_{Z}\left(y^{\prime}\right)}{H_{Z}(y)} e^{\Phi\left(y^{\prime}\right)-Z . r\left(y^{\prime}\right)-\beta}= & \frac{1}{H_{Z}(y)} e^{S_{n}(\phi)\left(\sigma^{-n}(y)\right)-n Z} \times \\
& \sum_{y^{\prime} \in g^{-1}\left(C_{-n, 0}(x)\right)} H_{Z}\left(y^{\prime}\right) e^{S_{r\left(y^{\prime}\right)-n}\left(y^{\prime}\right)-\left(r\left({ }^{\prime} y\right)-n\right) Z}
\end{aligned}
$$

Again,

$$
\sum_{y^{\prime} \in g^{-1}\left(C_{-n, 0}(x)\right)} H_{Z}\left(y^{\prime}\right) e^{S_{r\left(y^{\prime}\right)-n}\left(y^{\prime}\right)-\left(r\left({ }^{\prime} y\right)-n\right) Z}=\sum_{p=0}^{+\infty} L_{l}^{p}\left(H_{Z} \circ \sigma^{-1} e^{\phi \circ \sigma^{-1}-Z}\right)\left(\sigma^{-n}(y)\right),
$$

where $\sigma^{-1}\left(\left[\xi_{0}, \xi_{1}, \ldots\right]\right)$ means $\left[i_{R}, \xi_{0}, \xi_{1}, \ldots\right]$. We use (13) and conclude as above. Note that the constant in $\mathcal{O}$ only depends on $e^{\|\phi\|_{\infty}}$ and $\left\|H_{Z}\right\|_{\alpha}$ and $\|\phi\|_{\alpha}$. 
Now, if $\left[x_{-n}, \ldots, x_{-1}\right]$ is not an admissible word for any $\Sigma_{R}^{l}$, the same computation holds, except that the pressure of the component is strictly smaller than $Z_{c}$. It can thus be written under the form $Z_{c}-\varepsilon$ for some positive $\varepsilon$.

As there are finitely many irreducible components, we can find some uniform $\varepsilon$, independent of the components.

Now, note that $H_{Z}$ converges uniformly as $Z$ goes to $Z_{c}$. Moreover $\frac{e^{-\beta}}{1-e^{Z_{c}-Z}}$ converges to $\mathcal{A}$. Therefore, Lemma 4.1 and Lemma 4.2 yield that $\widehat{\mu}_{Z, y}^{s}\left(C_{-n, 0}^{1-e^{Z}}(x)\right)$ converges uniformly in $y$ as $Z$ goes to $Z_{c}$. This finishes the proof of the convergence of $\widehat{\mu}_{Z}$. Moreover, Lemma 3.1 and Lemma 4.1 prove that the limit measure $\widehat{\mu}$ only gives positive weight to the set of points whose orbit intersects $R$ only once. This set is totally dissipative with respect to $\sigma$.

Remark 8. Note that (24) yields that $\pi_{F} \widehat{\mu}=\mu:=\lim _{Z} \mu_{Z}$.

Remark 9. The measure $\widehat{\mu}_{Z}$ is $g$-invariant, but $g$ is not a continuous map. Therefore, there are no reason why the limit measure should be $g$-invariant. Nevertheless, we can consider it is the case, if we consider that for $\widehat{\mu}$-almost every point the return time (by iterations of $\sigma$ or $\sigma^{-1}$ ) is infinite.

We set $\mu^{\prime} \stackrel{\text { def }}{=} \sum_{k \geq 0} \sigma_{*}^{k} \widehat{\mu}$. It is a $\sigma$-finite measure and we now want to study its asymptotic with respect to $\sigma$.

Definition 4.3. Let $x$ be in supp $\mu^{\prime}$. There exists a unique integer $n$ such that $\sigma^{n}(x) \in R$. Then, there exists a unique $l$ such that for every $k \geq 0$, the cylinder $C_{n+1, n+k+1}(x)$ defines an admissible word for $\Sigma_{R}^{l}$. We say that $\Sigma_{R}^{l}$ (eventually) catches $x$.

Proposition 4.4. Let $f$ be a continuous functions in $\Sigma$. Then for $\mu^{\prime}$ almost every $x$,

$$
\lim _{n \rightarrow+\infty} \frac{1}{n} \sum_{j=0}^{n-1} f \circ \sigma^{j}(x)=\int f d m^{l(x)},
$$

where $m^{l(x)}$ is equilibrium state with respect to $\phi$ of the irreducible component of $\Sigma_{R}^{l(x)}$ which catches $x$.

Proof. Let us pick some irreducible component of $\Sigma_{R}$ with maximal $\phi$-pressure, $\Sigma_{R}^{l}$; $m^{l}$ is its equilibrium state. For $m^{l}$-almost every $z$ in $\Sigma_{R}^{l}$,

$$
\lim _{n \rightarrow+\infty} \frac{1}{n} S_{n}(f)(z)=\int f d m^{l} .
$$

As $f$ is continuous, if the limit occurs for some $z$, it also holds for every $z^{\prime}$ in its stable leaf. Moreover if the limit holds for $z$, it also holds for every $\sigma^{n}(z)$.

Now, remember that $m^{l}$ is defined on $\Sigma_{R}^{l}$, and its projection onto $\Sigma_{R}^{l+}$ is the measure defined by $\mathcal{H}_{l} . d \nu_{l}$ (see Subsection 2.1). Moreover $\mathcal{H}_{l}$ is bounded from below away to 0 and from above. 
Therefore, for $\nu^{l}$-almost every $z$ in $\Sigma_{R}^{l}{ }^{+}$, and for every $z^{\prime}$ in $W^{s}(z)$,

$$
\lim _{n \rightarrow+\infty} \frac{1}{n} S_{n}(f)\left(z^{\prime}\right)=\int f d m^{l} .
$$

We denote by $\Omega^{l}$ this set of full $\nu^{l}$-measure in $\Sigma_{R}{ }^{l+}$.

Now, Remark 8 says that the projection of $\widehat{\mu}$ onto $F$ in $R$ is $\mu$, the limit measure for $\mu_{Z}$. Remember that $d \mu_{Z}=H_{Z} d \nu_{Z}$ and $H_{Z}$ uniformly converges to $H$ and $\nu_{Z}$ converges to $\nu$. Moreover, $H_{Z}(\xi) \in\left[e^{-C_{\phi}}, e^{C_{\phi}}\right]$ for every $\xi$ in $F$, which means that $H$ is bounded from below away to 0 and from above. Hence, the projection of $\widehat{\mu}$ onto $F$ is equivalent to $\nu$.

For points which are caught by $\Sigma_{R}^{l}$, Proposition 3.6 yields that $\nu$ is equivalent to $\nu^{l} \circ \sigma^{-1}$. Therefore, for $\nu$-almost every $z$ in $F^{l}, \sigma(z)$ belongs to $W^{s}\left(\Omega^{l}\right)$. This means that for $\nu$-a.e. point in $F$ caught by $\Sigma_{R}^{l}$, the limit holds. Hence this also holds for $\widehat{\mu}$-a.e. point in $R$ caught by $\Sigma_{R}^{l}$. This finishes the proof.

\section{Proof of Theorem 2 and Corollary 3}

In this section, we construct a $\phi$-cstm which is related to $m$. We then prove that this system results from the disintegration of some $\sigma$-finite $\sigma$-invariant measure. Finally we prove that changing our choice for $R$, we have infinitely many different such measures.

\subsection{Construction of the $\phi$-cstm}

Following [BM77], the mixing property in $\Sigma$ proves that it is sufficient to construct one measure $\nu_{F}$ on $F$.

We want to prove that the measure we construct satisfies the property of being $\phi$-conformal, i.e., some strict property on the derivative of Radon-Nikodym for holonomies. It turns out that this property will be satisfied if we asked for another property, in relation with $\sigma$ and not the holonomy (see Remark 2).

We decompose $F$ in a disjoint union of sets $F_{n}, 0 \leq n \leq \infty$. A point $x$ in $F$ belongs to $F_{n}$ if and only if it returns $n$ times into $R$ by iterations of $\sigma$. Clearly $F_{\infty}$ is $g_{F}$-invariant. Note that $x$ belongs to $F_{n}$, if and only if $g_{F}^{n}(x)$ belongs to $F_{0}$. Therefore, if $x$ belongs to $F_{\infty}$, no points in its stable leaf $W^{s}(x)$ can be in $\bigcup_{n<+\infty} F_{n}$.

On $F_{0}$ we put the measure $\nu \stackrel{\text { def }}{=} \lim _{Z \rightarrow Z_{c}} \nu_{Z}$. Note that by Lemma $3.1 \mu\left(F_{0}\right)=1$, and we have seen that $\mu$ and $\nu$ are two equivalent probabilities. Points in $F_{0}$ are points whose forward orbit never returns into $R$, hence are points caught by some irreducible component. Therefore, $\nu$-almost very point belongs to some $F^{l}$, $l=1, \ldots, P$. Due to the density of the leaves, the pair $(F, \nu)$ is going to fix all the system of transverse measure. We however define $\nu_{F}$ everywhere on $F$ and will check later that this is coherent with the $\phi$-conformal property.

We decompose $F_{n}$ in disjoints $K_{n}$-sets, defined by the relation

$$
g_{F}^{n}\left(K_{n}(x)\right)=\pi_{F} \circ \sigma^{r^{n}(x)}\left(K_{n}(x)\right)=F .
$$


Note that the map $\pi_{F}$ defines a bijection from $\sigma^{r^{n}(x)}\left(K_{n}(x)\right)$ onto $F$. We push $\nu$ on $\sigma^{r^{n}(x)}\left(K_{n}(x)\right)$, following the $\phi$-conformal rule. We then define the measure $\nu_{F}$ on $K_{n}(x)$ following the rule

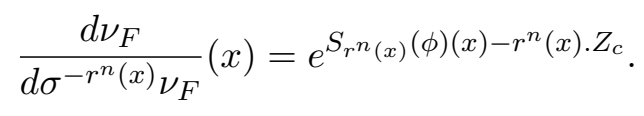

By construction, this measure is infinite because $\nu_{F}\left(F_{1}\right)=\mathcal{L}_{Z_{c}}(\mathbb{I})=+\infty$.

We now have to check that this definition of $\nu_{F}$ allows us to construct a unique $\phi$-conformal system of transversal measures on the local unstable leaves in $\Sigma$.

Let $A$ be any set in $F_{n}$. We assume it is included in a unique $K_{n}$-set, say $K_{n}(x)$. For any other $K_{n}$-set, $K_{n}(y)$, we can define the adjoint set $A^{\prime}$, in the following way:

Take the $g^{n}$-images of $K_{n}(x)$ and $K_{n}(y)$, project on $g^{n}\left(K_{n}(y)\right)$ the set $g^{n}(A)$ along the local stable leaves, and then take the preimage by $g^{n}$ of that set in $K_{n}(y)$.

Due to the definition of $\nu_{F}$ on the $K_{n}$-sets, the derivative of Radon-Nikodym of the image by that holonomy of $\nu_{F \mid K_{n}(x)}$ with respect to $\nu_{F \mid K_{n}(y)}$ is $e^{\omega}$.

Let us now take two $W^{s}$-conjugated sets $A$ and $A^{\prime}$ in different $F_{n}$ 's. By $W^{s}$ conjugated sets we means sets $A$ and $A^{\prime}$ such that there exists some bi-measurable holonomy $h^{s}$ from one to the other. Iterating by the map $\sigma$, we can always assume that one (namely $A$ ) is taken in $F_{n}$, and $A^{\prime}$ in $F_{0}$. We can also assume that $A^{\prime}$ is included in a unique $F^{l}$ and that $A$ is included in a unique $K_{n}$-set (otherwise we split each set in disjoints subsets with these properties).

Proposition 3.6 means that in $F_{0} \cap A^{\prime}$ the measure $\nu=\nu_{F}$ is the measure $a_{l} . \nu^{l} \circ \sigma$. Each $\nu^{l}$ is a conformal measure and satisfies in $\Sigma_{R}^{l+}$

$$
\frac{d \nu^{l}}{d \sigma^{-1} \nu^{l}}(x)=e^{\phi(x)-Z_{c}} .
$$

Note that this relation is equivalent to the one satisfied by $\nu^{l} \circ \sigma$ in $F^{l}$, stated in Proposition 3.6 and to (27). We can thus use the cocycle relation. Let $p$ be such that $g^{n}(A)=\sigma^{p}(A)$. The $W^{s}$-conjugacy between $A$ and $A^{\prime}$ is then equivalent to the $W^{s}$-conjugacy between $\sigma^{p}(A)$ and $\sigma^{p}\left(A^{\prime}\right)$. Moreover $\sigma^{p}(A)$ belongs to $R$, and the future of $\sigma^{p}(A)$ belongs to $\Sigma_{R}^{l+}$.

Then, Proposition 3.6, equality (28) and the definition of $\nu_{F}$ on $F_{n}$ imply that the derivative of Radon-Nikodym between $\nu_{F}$ and $h_{*}^{s} \nu_{F}$ for any point $x$ in $A$ is $e^{\omega}$. This thus proves that the $\phi$-conformal property holds for this choice of $W^{s}$-conjugated sets $A$ and $A^{\prime}$ in $F$. Following [BM77] we can extend $\nu_{F}$ using the $\phi$-conformal property to get some $\phi$-cstm in $\Sigma$.

Remark 10. Note that for every local unstable leaf in $\Sigma_{R}^{l}$, the restriction of the measure defined by the system above is equivalent to $\nu^{l}$.

We also want to point-out that the system of measures we have just defined satisfies the property:

$$
\frac{d \mu_{x}}{d \sigma^{-1} \mu_{\sigma(x)}}(x)=e^{\phi(x)-Z_{c}} .
$$

This follows from the definition of $\nu_{F}$ in $F$ and of $\nu$ in $F_{0}$. 


\subsection{Integration of the system of measures}

Lemma 5.1. The measure $\widehat{\mu}$ has a product structure: $\widehat{\mu} \equiv \nu \otimes \nu^{s}$, for some probability measure $\nu^{s}$. The measure $\nu^{s}$ only weights points in $R$ which never return into $R$ by iterations of $\sigma^{-1}$.

Proof. First, Lemma 4.1 proves that $\widehat{\mu}$ gives positive weight only to sets of points in $R$ whose backward orbit never intersects $R$ again. Let us pick such a point $x$. Therefore its past $\left[\ldots, x_{-2}, x_{-1}\right]$ is an admissible word for a unique irreducible component of $\Sigma_{R}$. Lemma 4.2 yields that $\widehat{\mu}$ gives positive weight only to sets of points $x$ such that $\left[\ldots, x_{-2}, x_{-1}\right]$ is an admissible word for one of the $\Sigma_{R}^{l}$. Let us assume in addition that this holds for $x$. Equalities (26) and (24) yield

$$
\begin{aligned}
\widehat{\mu}\left(C_{-n, m}(x)\right) & =\int H \cdot e^{\phi-Z} d \nu^{l} \circ \sigma \times \\
& \int \mathcal{H}_{l}\left(\sigma^{-n}(y)\right) e^{S_{n}(\phi)\left(\sigma^{-n}(y)\right)-n Z_{c}} \mathbb{I}_{C_{0, m}(x)}\left(\pi_{F}(y)\right) \mathbb{I}_{C_{-n, 0}(x)}(y) d \nu\left(\pi_{F}(y)\right),
\end{aligned}
$$

This gives

$$
\frac{\widehat{\mu}\left(C_{-n, m}(x)\right)}{\widehat{\mu}\left(C_{-n, 0}(x)\right)}=\frac{\int \mathcal{H}_{l}\left(\sigma^{-n}(y)\right) e^{S_{n}(\phi)\left(\sigma^{-n}(y)\right)} \mathbb{I}_{C_{-n, 0}(x)}(y) \mathbb{I}_{C_{0, m}(x)}\left(\pi_{F}(y)\right) d \nu\left(\pi_{F}(y)\right)}{\int \mathcal{H}_{l}\left(\sigma^{-n}(y)\right) e^{S_{n}(\phi)\left(\sigma^{-n}(y)\right)} \mathbb{I}_{C_{-n, 0}(x)}(y) d \nu\left(\pi_{F}(y)\right)} .
$$

We know (see [Roh62]) that for $\widehat{\mu}$-a.e. point $x$, the limit in the left hand side of (30) exists as $n$ goes to $+\infty$, and equals $\widehat{\mu}_{x}^{u}\left(C_{0, m}(x)\right)$. We also note that the right hand term in (30) belongs to $\left[\nu\left(C_{0, m}(x)\right) e^{-4 C_{\phi}}, \nu\left(C_{0, m}(x)\right) e^{4 C_{\phi}}\right]$. Indeed, each $\mathcal{H}_{l}(\xi)$ belongs to $\left[e^{-C_{\phi}}, e^{C_{\phi}}\right]$, and each term $e^{S_{n}(\phi)\left(\sigma^{-n}(y)\right)}$ can be replaced, up to $e^{ \pm C_{\phi}}$ by $e^{S_{n}(\phi)\left(\sigma^{-n}(\xi)\right)}$ for a fixed $\xi$ in the same cylinder.

This means that for $m$ and for every $x$,

$$
e^{-4 C_{\phi}} \leq \frac{\widehat{\mu}_{x}^{u}\left(C_{0, m}(x)\right)}{\nu\left(C_{0, m}(x)\right)} \leq e^{4 C_{\phi}} .
$$

Hence, the conditional measure $\widehat{\mu}_{x}^{u}$ is equivalent to $\nu$. This effectively proves that $\widehat{\mu}$-almost everywhere, the conditional measures are equivalent (up to the stable holonomy which exactly is the horocycle flow). This proves that $\widehat{\mu}$ has a product structure.

We can thus set $\widehat{\mu} \equiv \nu \otimes \nu^{s}$, for some probability measure $\nu^{s}$. Note that $\nu^{s}$ only weights points in $R$ which never return into $R$ by iterations of $\sigma^{-1}$ because $\widehat{\mu}$ does.

Let us set

$$
\widetilde{\tau} \stackrel{\text { def }}{=} \nu_{F} \otimes \nu^{s} .
$$

Then $\widetilde{\tau}$ only weights points in $R$ which never return into $R$ by iterations of $\sigma^{-1}$ and which return only finitely many times in $R$ (possibly 0 ) by iterations of $\sigma$. This set is totally dissipative. Let us set

$$
\tau \stackrel{\text { def }}{=} \sum_{k \in \mathbb{Z}} \widetilde{\tau} \circ \sigma^{k}
$$


which is a $\sigma$-finite and $\sigma$-invariant measure. By construction it integrates the $\phi$ conformal system of measures defined by $\nu_{F}$ in $\Sigma$. It is non ergodic, because it weights points whose orbits intersects $R$ only finitely many times; this set can be decomposed in $\sigma$-invariant disjoints sets, defined as the set of points whose orbit intersect $R$ exactly $n$-times, $n$ describing $\mathbb{N}^{*}$. All these sets have positive measure.

We now check that the measure $\tau$ is $\sigma$-finite. For that we only need to check that $\widetilde{\tau}$ is $\sigma$-finite. We can decompose the support of $\widetilde{\tau}$ in sets of points in $\pi_{F}^{-1}\left(F_{n}\right)$. Moreover, each $\pi_{F}^{-1}\left(F_{n}\right)$ can be decomposed in the disjoint and countable union of $\pi_{F}^{-1}\left(K_{n}\right)$-sets. Each such set has finite $\widetilde{\tau}$-measure because its image by $g^{n}$ is into $\pi_{F}^{-1}\left(F_{0}\right)$. This finishes the construction and the proof of Theorem 2 .

\subsection{Proof of corollary 3: counting these measures}

There are only finitely vertices's in $\Sigma$, but it is possible to use a higher block representation (see [LM95]) to increase this number. Now, using the higher block representation, we can chose $R^{\prime} \subset R$ (and $R^{\prime} \neq R$ ). Obviously, points which only return finitely many times in $R$ by iterations of $\sigma$ also only return finitely many times in $R^{\prime}$. However the new $\phi$-cstm, $\left\{\mu_{W_{l o c}^{u}}^{\prime}\right\}$, satisfies

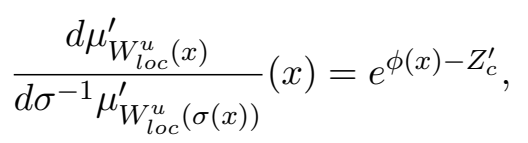

where $Z_{C}^{\prime}$ is the pressure of the dotted system with hole $R^{\prime}$. Note that in $\Sigma, R^{\prime}$ is a cylinder with some length $n$. Making this length $n$ go to $+\infty$, the pressure of the dotted system has to increase to the pressure of $\Sigma$ (but never equals it). Therefore it attempts infinitely many different values. Hence (29) and (31) prove that all these systems are different, thus all their integrated measures are different. It effectively furnishes infinitely many different measures as announced. Corollary 3 is proved.

\section{References}

[Aar97] J. Aaronson. An introduction to infinite ergodic theory, volume 50 of Mathematical Surveys and Monographs. American Mathematical Society, Providence,RI, 1997.

[Ale76] V. M. Alekseyev. Symbolic dynamic. In 11th summer mathematical school. Mathematics Institute of the Ukraininan Academy of Sciences, Kiev, 1976.

[Arn68] Leslie K. Arnold. On $\sigma$-finite invariant measures. Z. Wahrscheinlichkeitstheorie und Verw. Gebiete, 9:85-97, 1968.

[Bal00] V. Baladi. Positive transfer operators and decay of correlations, volume 16 of Advanced Series in Nonlinear Dynamics. World Scientific, 2000.

[BM77] R. Bowen and B. Marcus. Unique Ergodicity for Horocycle Foliations. Israel Journal of Mathematics, 26(1):43-67, 1977.

[Bow75] R. Bowen. Equilibrium States and the Ergodic Theory of Anosov Diffeomorphisms, volume 470 of Lecture notes in Math. Springer-Verlag, 1975. 
[Bur90] M. Burger. Horocycle flow on geometrically finite surfaces. Duke Math. J., 61(3):779-803, 1990.

[CL05] J.-R. Chazottes and R. Leplaideur. Fluctuations of the N th return time for Axiom A diffeomorphisms. Discrete Contin. Dyn. Syst., 13(2):399-411, 2005 .

[CLT01] G. Contreras, A. Lopes, and P. Thieullen. Lyapunov minimizing measures for expanding maps of the circle. Ergod. Th. E Dynam. Sys., 21:1-31, 2001.

[Cou01] Y. Coudene. Cocycles and stable foliations of Axiom A flows. Ergodic Theory Dynam. Systems, 21(3):767-775, 2001.

[Dan78] S. G. Dani. Invariant measures of horospherical flows on noncompact homogeneous spaces. Invent. Math., 47(2):101-138, 1978.

[Fur73] H. Furstenberg. The unique ergodicity of the horocycle flow. In Recent advances in topological dynamics (Proc. Conf., Yale Univ., New Haven, Conn., 1972; in honor of Gustav Arnold Hedlund), pages 95-115. Lecture Notes in Math., Vol. 318. Springer, Berlin, 1973.

[GLT10] E. Garibaldi, A.O. Lopes, and Ph. Thieullen. On calibrated ans separating sub-actions. Boletim da Sociedade Brasileira de matemática, to appear:????, 2010.

[Hay94] N. T.A. Haydn. Canonical product structure of equilibrium states. Random and computational dynamics, 2(1):79-96, 1994.

[Lep00] R. Leplaideur. Local product structure for equilibrium states. Trans. Amer. Math. Soc., 352(4):1889-1912, 2000.

[Lep05] R. Leplaideur. A dynamical proof for the convergence of Gibbs measures at temperature zero. Nonlinearity, 18(6):2847-2880, 2005.

[LM95] D. Lind and B. Marcus. An introduction to Symbolic Dynamics and Coding. Cambridge University Press, 1995.

[LS07] F. Ledrappier and O. Sarig. Invariant measures for the horocycle flow on periodic hyperbolic surfaces. Israel Journal of Mathematics, 160:281-315, 2007.

[Roh62] V.A. Rohlin. On the fundamental ideas of measure theory. A.M.STranslation, 10(1):1-52, 1962.

[Sch77] K. Schmidt. Infinite invariant measures on the circle. In Symposia Mathematica, Vol. XXI (Convegno sulle Misure su Gruppi e su Spazi Vettoriali, Convegno sui Gruppi e Anelli Ordinati, INDAM, Rome, 1975), pages 3743. Academic Press, London, 1977. 